\title{
Long-range spin transport on the surface of topological Dirac semimetal
}

\author{
Yasufumi Araki $\odot,{ }^{1}$ Takahiro Misawa $\odot,{ }^{2}$ and Kentaro Nomura ${ }^{3,4}$ \\ ${ }^{1}$ Advanced Science Research Center, Japan Atomic Energy Agency, Tokai 319-1195, Japan \\ ${ }^{2}$ Beijing Academy of Quantum Information Sciences, Haidian District, Beijing 100193, China \\ ${ }^{3}$ Institute for Materials Research, Tohoku University, Sendai 980-8577, Japan \\ ${ }^{4}$ Center for Spintronics Research Network, Tohoku University, Sendai 980-8577, Japan
}

(Received 30 March 2021; accepted 19 May 2021; published 21 June 2021)

\begin{abstract}
We theoretically propose the long-range spin transport mediated by the gapless surface states of a topological Dirac semimetal (TDSM). Low-dissipation spin current is a building block of next-generation spintronics devices. While conduction electrons in metals and spin waves in ferromagnetic insulators (FMIs) are the major carriers of spin current, their propagation length is inevitably limited due to Joule heating or Gilbert damping. In order to suppress dissipation and realize long-range spin transport, we here make use of the spin-helical surface states of TDSMs, such as $\mathrm{Cd}_{3} \mathrm{As}_{2}$ and $\mathrm{Na}_{3} \mathrm{Bi}$, which are robust against disorder. Based on a junction of two FMIs connected by a TDSM, we demonstrate that the magnetization dynamics in one FMI induces a spin current on the TDSM surface flowing to the other FMI. By both the analytical transport theory on the surface and the numerical simulation of real-time evolution in the bulk, we find that the induced spin current takes a universal semiquantized value that is insensitive to the microscopic coupling structure between the FMI and the TDSM. We show that this surface spin current is robust against disorder over a long range, which indicates that the TDSM surface serves as a promising system for realizing spintronics devices.
\end{abstract}

DOI: 10.1103/PhysRevResearch.3.023219

\section{INTRODUCTION}

Transmission of signals over a long distance is essential in designing integrated information devices. While charge current in normal metals is inevitably subject to Joule heating, spin current is now intensely studied to realize long-range transmission with less dissipation, in the context of spintronics [1-3]. Spin current, namely, the flow of spin angular momentum, is carried by various types of quasiparticle excitations in materials. In metals, spin current is carried by conduction electrons [4]. Electron spin current can be generated by current injection from magnetic metals [5], spin pumping from magnetic materials with magnetization dynamics [6-8], the spin Hall effect [9-13], etc. Spin waves (or magnons) in magnetic materials, namely, the dynamics of the ferromagnetic or antiferromagnetic order parameters, are also elementary excitations that carry spin and heat currents [14-18]. Magnon spin current can be generated by the magnetic resonance under a microwave $[19,20]$, by the spin Seebeck effect under a temperature gradient $[21,22]$, etc.

While those spin-current carriers are available in various materials commonly used in experiments, their propagation length is inevitably limited due to dissipation. Conduction electrons are subject to scattering by phonons and disorder,

Published by the American Physical Society under the terms of the Creative Commons Attribution 4.0 International license. Further distribution of this work must maintain attribution to the author(s) and the published article's title, journal citation, and DOI. which results in Joule heating. Magnon spin current in insulators is considered to be advantageous in that it is free from Joule heating [23-26], whereas Gilbert damping of spins leads to the dissipation of spin and energy. Due to such dissipation effects, transmission of spin current over a long range is a challenging problem. Recent theoretical and experimental studies showed that phonons in nonmagnetic insulators may realize long-range transport of spin current [27-32]: Since circularly polarized transverse phonons carry angular momentum, they can mediate spin current between magnets via magnetoelastic coupling. However, for the efficient interconversion of magnons and phonons, one needs fine-tuning of the magnon frequency. Such limitations in long-range spin transport restrict the design of highly integrated spintronics devices.

In this paper, we theoretically propose a long-range transmission of spin mediated by the surface electronic states of topological Dirac semimetals (TDSMs). TDSMs are a class of three-dimensional (3D) crystalline materials having a pair (or pairs) of Dirac nodes in the electronic band structure in the bulk [33-35]. The TDSM phase is experimentally realized in $\mathrm{Na}_{3} \mathrm{Bi}$ [36,37] and $\mathrm{Cd}_{3} \mathrm{As}_{2}$ [38-43]. TDSMs have quasi-1D gapless states on the surface, which arise as Fermi arcs connecting the Dirac points projected onto the surface Brillouin zone $[44,45]$. These surface states are spin helical, i.e., spin- $\uparrow$ and spin- $\downarrow$ states propagate along the surface oppositely to each other, and are protected by the $\mathbb{Z}_{2}$ topology in the bulk [46,47]. These features are analogous to the helical edge states of 2D quantum spin Hall insulators (QSHIs) [48-50], and hence the surface states of TDSMs are robust against disorder as long as the system preserves time-reversal 


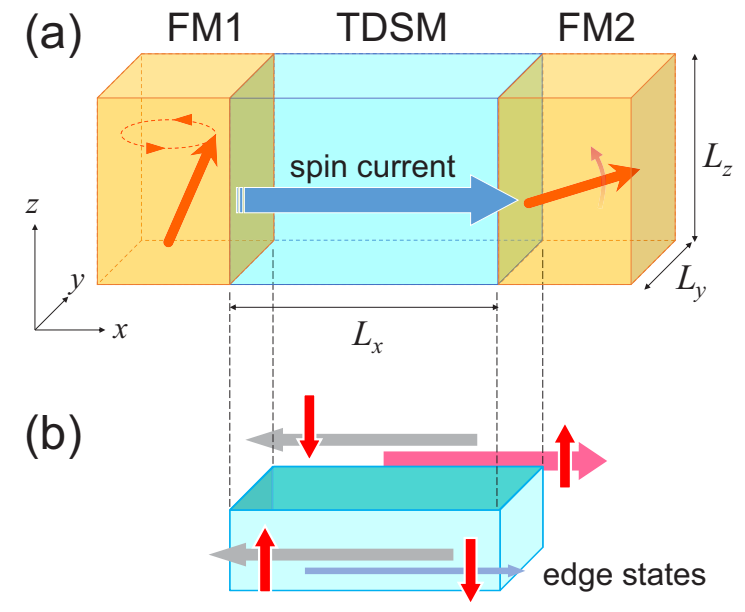

FIG. 1. (a) Schematic illustration of our setup for a joint system between a topological Dirac semimetal (TDSM) and ferromagnetic insulators (FMIs). The magnetization dynamics in the left-side FMI (FM1) induces a spin current flowing through the TDSM. We investigate the torque exerted on the right-side FMI (FM2). (b) Schematic illustration of the spin current carried by the helical surface states of the TDSM. There are four spin-polarized channels on the surface of the TDSM connecting FM1 and FM2. The magnetization dynamics induces population imbalance among the four edge channels, which yields a net spin current from FM1 to FM2.

symmetry [51,52]. From these features, we can expect that the helical surface states of TDSMs are suitable for long-range spin transmission.

Indeed, in the context of 2D QSHIs, it was theoretically proposed in numerous studies in the literature that the helical edge states are capable of interconversion between spin angular momentum and electric current, based on the topological field theory, the numerical simulations, the scattering theory, and the Floquet theory [53-60]. The electric current and the spin torque arising from the interconversion take quantized values irrespective of the microscopic coupling structure between the edge electrons and the spins in magnets, which is traced back to the band topology of the 1D edge states. The similar spin-charge interconversion is expected also on the helical surface states of TDSMs, as long as the Fermi level is tuned in the vicinity of the Dirac points so that the bulk transport may be negligible [61]. However, the spin-charge conversion discussed in those works occurs locally at the interface of a magnet and a TDSM (or a QSHI), and a theory for nonlocal transmission of spins with the helical edge states over a long distance, which is essential for device application, is not well established.

Based on the above background, we here consider the transmission of spin angular momentum between two ferromagnetic insulators (FMIs) connected by a TDSM, as schematically shown in Fig. 1(a). We assume a magnetization dynamics in one of the FMIs (FM1) and discuss how the spin current transmitted through the TDSM exerts a spin torque on the other FMI (FM2), by constructing analytical and numerical schemes to evaluate the nonlocal spin transmission between the two FMIs separated at a distance. As a result, we find that the transmitted spin current takes a semiquantized value, which is determined only by the configuration of Dirac points in momentum space and the frequency of magnetization dynamics. This semiquantization of spin current can be understood analytically as the electron transport on the helical surface states, which comes from the imbalance of the electron population among the edge channels driven by the magnetization dynamics [see Fig. 1(b)]. Moreover, from the numerical simulation of the real-time dynamics of electrons in the whole 3D system, we directly confirm that this semiquantized spin transmission is robust against moderate disorder in the bulk. These results imply that the TDSM surface may serve as a promising system for highly integrated spintronics devices with long-range spin transmission.

This paper is organized as follows. In Sec. II, we review the generic characteristics of TDSMs and give a detailed explanation about our model setup with a TDSM and FMIs shown in Fig. 1. In Sec. III, we give analytical expressions of the flow of electrons and spin on the surface, based on the 1D scattering theory. (The detailed calculation processes are shown in Appendix.) In Sec. IV, we show the results of our numerical simulations within the whole 3D system and discuss their consistency with the analytical expressions and their robustness against disorder. Finally, in Sec. V, we give some experimental implications from our calculations and conclude our discussion. Throughout this paper, we take the natural unit $\hbar=1$.

\section{MODEL SETUP WITH A TDSM}

In order to demonstrate spin transmission through a TDSM, we construct a model that we shall use both for the analysis and for the numerical simulation, as shown in Fig. 1. We first review the generic characteristics of TDSMs and give a detailed explanation about our model setup, with a junction of a TDSM and two FMIs, on the basis of those characteristics.

TDSMs are characterized by a pair of Dirac points in momentum space, which are protected by rotational symmetry around a crystal axis $[33,46,47]$. If we take this axis as the $z$ axis, the Dirac points are located on the $k_{z}$ axis, which we denote as $\boldsymbol{k}_{D}^{ \pm}=\left(0,0, \pm k_{D}\right)$. Due to the rotational symmetry, the spin component $\sigma_{z}$ serves as a good quantum number around the $k_{z}$ axis, which means that the spin- $\uparrow$ and spin- $\downarrow$ states are degenerate around the Dirac points. The minimal model for such a band structure at low energy is composed of four degrees of freedom, with twofold spins and twofold orbitals [34,36,38],

$$
\begin{aligned}
H(\boldsymbol{k}) & =v\left(k_{x} \tau_{x} \sigma_{z}+k_{y} \tau_{y}\right)-M\left(k_{z}\right) \tau_{z}, \\
M\left(k_{z}\right) & =m_{0}-m_{1} k_{z}^{2} .
\end{aligned}
$$

Here, the Pauli matrices $\sigma_{x, y, z}$ act on the spin subspace, and $\tau_{x, y, z}$ act on the orbital subspace. In the effective model of $\mathrm{Cd}_{3} \mathrm{As}_{2}$ [38], for instance, the basis functions with $\tau_{z}=+$ correspond to the $5 s$ orbitals of $\mathrm{Cd}$ with the total angular momentum $J_{z}= \pm 1 / 2$, while those with $\tau_{z}=-$ correspond to the $4 p$ orbitals of As with $J_{z}= \pm 3 / 2$, and $\sigma_{z}= \pm$ represents the sign of $J_{z}$. The parameter $m_{0}$ characterizes the band inversion, which leads to the Dirac points of $k_{D}=\sqrt{m_{0} / m_{1}}$ if $m_{0}, m_{1}>0$. 


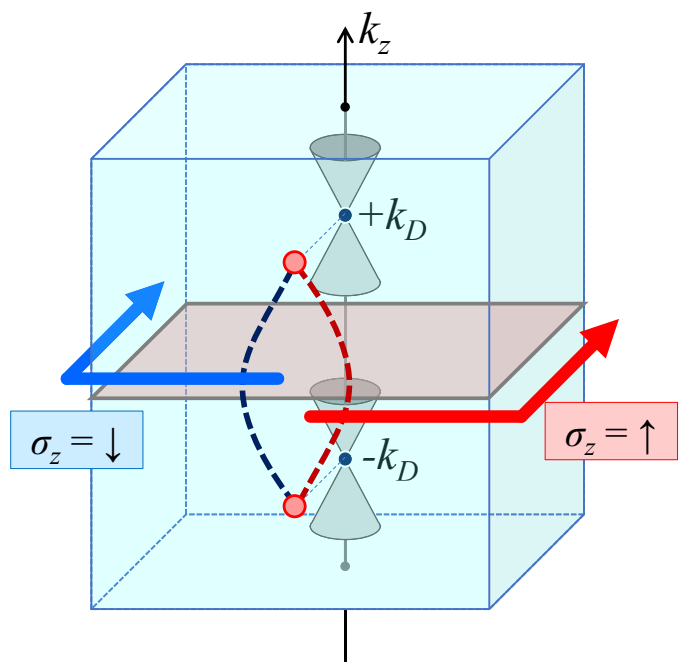

FIG. 2. Schematic picture of the structure of the surface states of TDSM. The surface states arise as Fermi arcs connecting the Dirac points projected onto the surface Brillouin zone, shown as dark red and blue dashed curves. They form a pair of counterpropagating states, one with spin $\sigma_{z}=\uparrow$ and the other with $\sigma_{z}=\downarrow$. Taking the 2D slice in the band-inverted region $\left(k_{z} \in\left[-k_{D}, k_{D}\right]\right.$, shown by the gray plane), the Hamiltonian reduces to that of the 2D QSHI.

Due to the band inversion from spin-orbit coupling, the system shows the intrinsic spin Hall effect. By fixing $k_{z}$ in the band-inverted region $-k_{D}<k_{z}<k_{D}$ and considering the 2D slice, as shown in Fig. 2, the Hamiltonian reduces to that for the 2D QSHI, with the quantized spin Hall conductivity $\sigma_{x y}^{s(2 \mathrm{D})}=e / 2 \pi$. Therefore, by multiplying by the number of $2 \mathrm{D}$ slices $v_{z}=2 k_{D} / 2 \pi$ per unit length in the $z$ direction, the spin Hall conductivity of the TDSM in 3D takes the "semiquantized" value

$$
\sigma_{x y}^{s(3 \mathrm{D})}=v_{z} \sigma_{x y}^{s(2 \mathrm{D})}=\frac{e k_{D}}{2 \pi^{2}},
$$

if the Fermi level is in the vicinity of the Dirac points [34,35].

Another consequence of the band inversion is the emergence of surface states. On the surfaces parallel to the rotational axis ( $z$ axis), which we call the side surfaces, there emerge spin-helical states, with the spin- $\uparrow$ and spin- $\downarrow$ modes propagating along the surface oppositely to each other [44]. These surface states can be regarded as the collection of 1D helical edge states of the 2D QSHI at fixed $k_{z}$. They form a pair of Fermi arcs connecting the Dirac points projected onto the surface Brillouin zone, which are robust against disorder as long as time-reversal symmetry is preserved [52]. The contribution of these surface states to the electron transport was observed experimentally, as the quantum oscillation under a magnetic field [40,51,62-67].

In order to make use of the helical surface states for spin transmission, here we consider the model setup as shown in Fig. 1, with two FMIs (FM1 and FM2) attached on the side surfaces of the TDSM. FM1 and FM2 are set apart by the distance $L_{x}$, and each of them is attached to the TDSM by the length $L_{y}$. We assume a situation where the magnetization of FM1 is steadily precessing around the $z$ axis, which is maintained by providing angular momentum and energy
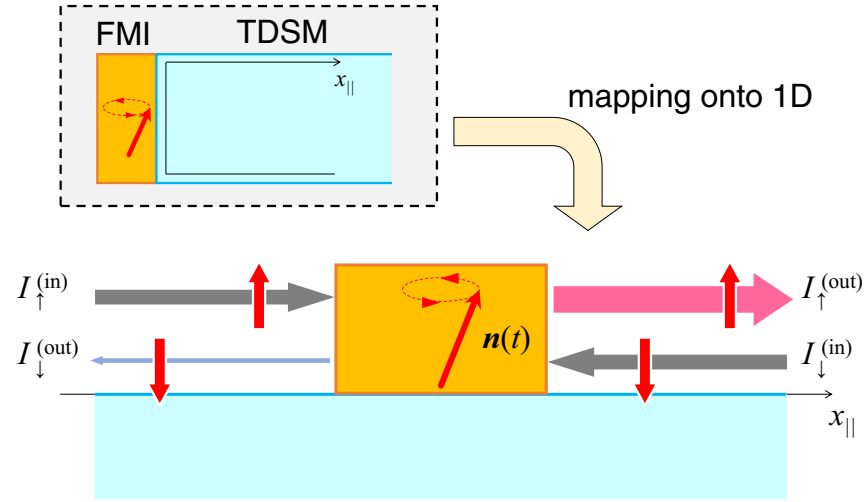

FIG. 3. Schematic picture for the pumping process of the edge electrons by a single FMI. The pumping process is mapped to the scattering problem in the quasi-1D space $\left(x_{\|}\right)$along the edge, by regarding the interface region with the precessing magnetization $\boldsymbol{n}(t)$ as the time-dependent scatterer. By solving the scattering problem as described in Sec. III B, we see that the outgoing channel with spin $\uparrow$ becomes more populated than that with spin $\downarrow$.

externally by microwaves, etc. Under such a setup, we estimate the spin torque exerted on FM2, which corresponds to the spin current transmitted from FM1 to FM2 via the TDSM, both analytically and numerically.

\section{TRANSPORT ANALYSIS ON THE SURFACE}

In this section, we treat the spin transmission through the TDSM analytically, by focusing on the spin transport mediated by the helical surface states. If the Fermi level is in the vicinity of the Dirac points, the bulk transport becomes negligible, and the surface transport becomes dominant. In order to evaluate the spin transmission between two FMIs phenomenologically, we first formulate the transmission of charge and spin at a single interface with a FMI. By using this single-FMI picture as a building block, we formulate the spin transmission between the two FMIs in our model setup shown in Fig. 1.

As mentioned in the previous section, we regard the helical surface states of the TDSM as the collection of 1D helical edge states, which reside at every $k_{z}$ in the band-inverted region $\left(-k_{D}<k_{z}<k_{D}\right)$. As long as the translational symmetry in the $z$ direction is satisfied, $k_{z}$ serves as a good quantum number, and the contribution from 1D helical edge states at each $k_{z}$ can be treated separately. Therefore, in this section, we first consider the spin transmission by a single pair of 1D helical edge states and then multiply by the number of 2D slices $v_{z}=2 k_{D} / 2 \pi$ per unit length in the $z$ direction to evaluate the overall contribution from the $2 \mathrm{D}$ surface states.

\section{A. Charge and spin pumping by a single FMI}

In a manner similar to the theoretical treatment of spin pumping and injection by a ferromagnet $[7,8]$, we formulate the role of a FMI coupled to the helical edge as a timedependent scatterer. We consider the scattering process in the hypothetical 1D space along the edge, where we denote its 1D coordinate as $x_{\|}$(see Fig. 3). 
As in the conventional Landauer-Büttiker formalism in mesoscopic systems [68-72], the charge and spin currents can be derived by comparing the numbers of the incoming and outgoing electrons for the scatterer. Since the nonmagnetic edge states are spin helical, there are two incoming channels and two outgoing channels: As the incoming channels, spin- $\uparrow$ electrons come from the left $\left(x_{\|}<0\right)$, and spin- $\downarrow$ electrons come from the right $\left(x_{\|}>0\right)$; as the outgoing channels, spin$\uparrow$ electrons go out to the right $\left(x_{\|}>0\right)$, and spin- $\downarrow$ electrons go out to the left $\left(x_{\|}<0\right)$. We denote the annihilation (creation) operators of an electron with energy $\epsilon$ in these channels as $a_{\uparrow / \downarrow}^{(\dagger)}(\epsilon)$ for the incoming channels and $b_{\uparrow / \downarrow}^{(\dagger)}(\epsilon)$ for the outgoing channels, respectively.

The electron distributions in these channels with energy $\epsilon$ are given by taking the quantum average $\langle\cdot\rangle$ of the operators defined above [69-71],

$$
f_{\uparrow / \downarrow}^{\text {(in) }}(\epsilon)=\left\langle a_{\uparrow / \downarrow}^{\dagger}(\epsilon) a_{\uparrow / \downarrow}(\epsilon)\right\rangle, \quad f_{\uparrow / \downarrow}^{\text {(out) }}(\epsilon)=\left\langle b_{\uparrow / \downarrow}^{\dagger}(\epsilon) b_{\uparrow / \downarrow}(\epsilon)\right\rangle,
$$

which we use throughout this section as the main tool to evaluate the transmission of spin current. With these distribution functions, the numbers of electrons coming into or going out of the scatterer with spin $\uparrow$ or $\downarrow$ per unit time are given by

$$
I_{\uparrow / \downarrow}^{\text {(in/out) }}=\frac{1}{2 \pi} \int d \epsilon f_{\uparrow / \downarrow}^{\text {(in/out) }}(\epsilon) .
$$

By using these notations, the charge current flowing from the left to the right is given by

$$
I=-e\left[I_{\uparrow}^{\text {(in) }}-I_{\downarrow}^{\text {(out) }}\right]=-e\left[I_{\uparrow}^{\text {(out) }}-I_{\downarrow}^{\text {(in) }}\right] .
$$

The two formalisms are equivalent due to the charge conservation at the scatterer. On the other hand, spin can be transferred between the electrons and the FMI, and thus the net spin current flowing out of the scatterer can be nonzero. Noting that each electron carries spin $\pm 1 / 2$, the spin current pumped by the FMI, namely, the net spin angular momentum flowing into and out of the FMI per unit time, is given by

$$
I^{s}=\frac{1}{2}\left[I_{\uparrow}^{\text {(out) }}-I_{\downarrow}^{\text {(out) }}-I_{\uparrow}^{\text {(in) }}+I_{\downarrow}^{\text {(in) }}\right] .
$$

From these relations, we can immediately see a simple relation between the charge and spin currents,

$$
\frac{1}{e} I+I^{s}=-I_{\uparrow}^{(\text {in })}+I_{\downarrow}^{(\text {in })},
$$

where the right-hand side is determined only by the numbers of incoming particles and is independent of the scattering process. In particular, if the numbers of spin- $\uparrow$ and spin- $\downarrow$ electrons entering the scattering region are equal, its right-hand side vanishes and reduces to the simple relation $I^{s}=-I / e$.

In order to evaluate the charge current $I$ and the spin current $I^{S}$ separately, we need relations between the incoming and outgoing distributions that are determined by the scatterer. If the magnetization $\boldsymbol{n}$ is periodically precessing as

$$
\boldsymbol{n}(t)=(\sin \theta \cos (\Omega t+\phi), \sin \theta \sin (\Omega t+\phi), \cos \theta),
$$

where $\Omega$ is the precession frequency and $\theta$ is the polar angle of magnetization, the energy of the electron is not conserved in the scattering process. Such a time-dependent scattering problem can be solved by taking the "rotating frame" of spin: By the time-dependent unitary transformation

$$
U(t)=e^{i \Omega t \sigma_{z} / 2}
$$

on the edge electrons, which rotates their spin by the angle $\Omega$ per unit time around the $z$ axis, the magnetization direction is fixed to $\boldsymbol{n}_{0} \equiv \boldsymbol{n}(t=0)$ in this rotating frame [57]. Since this transformation $U(t)$ shifts the energies of spin- $\uparrow$ or spin- $\downarrow$ electrons by $\pm \Omega / 2$, respectively, the operators in the rotating frame, which we denote by $\tilde{a}_{\uparrow / \downarrow}$ and $\tilde{b}_{\uparrow / \downarrow}$, are related to those in the rest frame as

$$
\begin{array}{ll}
\tilde{a}_{\uparrow}(\epsilon)=a_{\uparrow}\left(\epsilon+\frac{\Omega}{2}\right), & \tilde{a}_{\downarrow}(\epsilon)=a_{\downarrow}\left(\epsilon-\frac{\Omega}{2}\right), \\
\tilde{b}_{\uparrow}(\epsilon)=b_{\uparrow}\left(\epsilon+\frac{\Omega}{2}\right), & \tilde{b}_{\downarrow}(\epsilon)=b_{\downarrow}\left(\epsilon-\frac{\Omega}{2}\right) .
\end{array}
$$

The operators for the incoming and outgoing channels are related by the $S$ matrix. By using the $S$ matrix in the rotating frame

$$
\tilde{S}(\epsilon)=\left(\begin{array}{cc}
\tilde{r}_{\downarrow \uparrow}(\epsilon) & \tilde{t}_{\downarrow \downarrow}(\epsilon) \\
\tilde{t}_{\uparrow \uparrow}(\epsilon) & \tilde{r}_{\uparrow \downarrow}(\epsilon)
\end{array}\right),
$$

which can be obtained by solving the time-independent scattering problem with the fixed magnetization (see Appendix for details), the operators $\tilde{a}_{\uparrow / \downarrow}$ and $\tilde{b}_{\uparrow / \downarrow}$ in the rotating frame are related as [69-71]

$$
\left(\begin{array}{l}
\tilde{b}_{\downarrow}(\epsilon) \\
\tilde{b}_{\uparrow}(\epsilon)
\end{array}\right)=\left(\begin{array}{cc}
\tilde{r}_{\downarrow \uparrow}(\epsilon) & \tilde{t}_{\downarrow \downarrow}(\epsilon) \\
\tilde{t}_{\uparrow \uparrow}(\epsilon) & \tilde{r}_{\uparrow \downarrow}(\epsilon)
\end{array}\right)\left(\begin{array}{c}
\tilde{a}_{\uparrow}(\epsilon) \\
\tilde{a}_{\downarrow}(\epsilon)
\end{array}\right) .
$$

Note that the components in the $S$ matrix satisfy the reversibility relations

$$
\begin{aligned}
\left|\tilde{r}_{\downarrow \uparrow}(\epsilon)\right|^{2} & =\left|\tilde{r}_{\uparrow \downarrow}(\epsilon)\right|^{2} \equiv R(\epsilon), \\
\left|\tilde{t}_{\uparrow \uparrow}(\epsilon)\right|^{2} & =\left|\tilde{t}_{\downarrow \downarrow}(\epsilon)\right|^{2} \equiv T(\epsilon)
\end{aligned}
$$

and the unitarity condition

$$
R(\epsilon)+T(\epsilon)=1
$$

due to the time independence of the scatterer in the rotating frame, where $R(\epsilon)$ is the reflection rate and $T(\epsilon)$ is the transmission rate.

With the $S$ matrix defined above, we are ready to evaluate the electron distributions in the outgoing channels. By substituting Eqs. (10) and (12) into Eq. (3), we obtain the relations for the distribution functions in the rest frame as

$$
f_{\uparrow}^{\text {(out) }}\left(\epsilon+\frac{\Omega}{2}\right)=T(\epsilon) f_{\uparrow}^{\text {(in })}\left(\epsilon+\frac{\Omega}{2}\right)+R(\epsilon) f_{\downarrow}^{\text {(in })}\left(\epsilon-\frac{\Omega}{2}\right),
$$

$$
f_{\downarrow}^{\text {(out) }}\left(\epsilon-\frac{\Omega}{2}\right)=R(\epsilon) f_{\uparrow}^{\text {(in) }}\left(\epsilon+\frac{\Omega}{2}\right)+T(\epsilon) f_{\downarrow}^{\text {(in })}\left(\epsilon-\frac{\Omega}{2}\right) \text {, }
$$

which we shall use as the fundamental relations throughout this section to evaluate the spin transmission. By integrating over the energy $\epsilon$ and using the unitarity relation 
$R(\epsilon)+T(\epsilon)=1$, we obtain the relations between the numbers of incoming and outgoing electrons,

$$
\begin{aligned}
& I_{\uparrow}^{\text {(out) }}-I_{\uparrow}^{\text {(in) }}=\int \frac{d \epsilon}{2 \pi} R\left(\epsilon-\frac{\Omega}{2}\right)\left[f_{\downarrow}^{\text {(in) }}(\epsilon-\Omega)-f_{\uparrow}^{\text {(in })}(\epsilon)\right], \\
& I_{\downarrow}^{\text {(out) }}-I_{\downarrow}^{\text {(in) }}=\int \frac{d \epsilon}{2 \pi} R\left(\epsilon+\frac{\Omega}{2}\right)\left[f_{\uparrow}^{\text {(in) }}(\epsilon+\Omega)-f_{\downarrow}^{\text {(in) }}(\epsilon)\right] .
\end{aligned}
$$

\section{B. Scattering rates and quantized pumping}

The scattering rates $R(\epsilon)$ and $T(\epsilon)$ are defined in the rotating frame of spin, by a FMI with its magnetization fixed to the direction $\boldsymbol{n}_{0}$. The Hamiltonian for the edge electrons coupled to this magnetization in the rotating frame is given as

$$
\begin{aligned}
\tilde{H}\left(k_{\|}\right) & =v_{\text {edge }} k_{\|} \sigma_{z}+J \boldsymbol{n}_{0} \cdot \boldsymbol{\sigma}-\frac{\Omega}{2} \sigma_{z} \\
& =\left(\begin{array}{cc}
v_{\text {edge }} k_{\|}+J \cos \theta-\frac{\Omega}{2} & J \sin \theta e^{-i \phi} \\
J \sin \theta e^{i \phi} & -v_{\text {edge }} k_{\|}-J \cos \theta+\frac{\Omega}{2}
\end{array}\right),
\end{aligned}
$$

where we define the $1 \mathrm{D}$ momentum along the edge as $k_{\|}$. We can immediately see from this matrix form that the inplane component of the magnetization opens an exchange gap $J_{\perp} \equiv|J \sin \theta|$ around zero energy in the edge spectrum, which influences the scattering of the edge electrons by the FMI as we shall see in the following discussions.

By evaluating the $S$ matrix in the rotating frame, whose detailed derivation process is shown in Appendix, the scattering rates are given as

$$
\begin{aligned}
& R(\epsilon)=\frac{\sin ^{2}(K L)}{\epsilon^{2} / J_{\perp}^{2}-\cos ^{2}(K L)}, \\
& T(\epsilon)=\frac{\epsilon^{2} / J_{\perp}^{2}-1}{\epsilon^{2} / J_{\perp}^{2}-\cos ^{2}(K L)},
\end{aligned}
$$

with $K=\sqrt{\epsilon-J_{\perp}^{2}} / v_{\text {edge }}$ being the wave number inside the interface region coupled with the FMI. (Note that these forms are valid for $\epsilon$ inside the exchange gap, $|\epsilon|<J_{\perp}$, as well, where $K$ becomes pure imaginary and the wave function inside the interface region exponentially decays by $x_{\|}$.) The numerical behavior of $R(\epsilon)$ is shown in Fig. 4, by varying the electron energy $\epsilon$ and the length of the interface region $L$.

The most important feature in the reflection rate $R(\epsilon)$ is that it reaches unity for $|\epsilon|<J_{\perp}$, which means that the electron inside the exchange gap is totally reflected, if the length $L$ of the magnetic region is long enough. This is because the electron wave function in the magnetic region, at energies inside the exchange gap, decays exponentially. The decay length of the wave function at $\epsilon=0$ is given as

$$
l_{0}=\left[\operatorname{Im} K_{\epsilon=0}\right]^{-1}=v_{\text {edge }} / J_{\perp},
$$

and hence the tunneling through the magnetic region is fully suppressed if $L \gg l_{0}$. On the other hand, if $\epsilon$ is out of the exchange gap, the reflection rate $R(\epsilon)$ oscillates as a function of $\epsilon$ due to the formation of resonance states inside the interface region.

By using the scattering rates obtained above, we can evaluate the charge and spin currents pumped by the FMI. If we
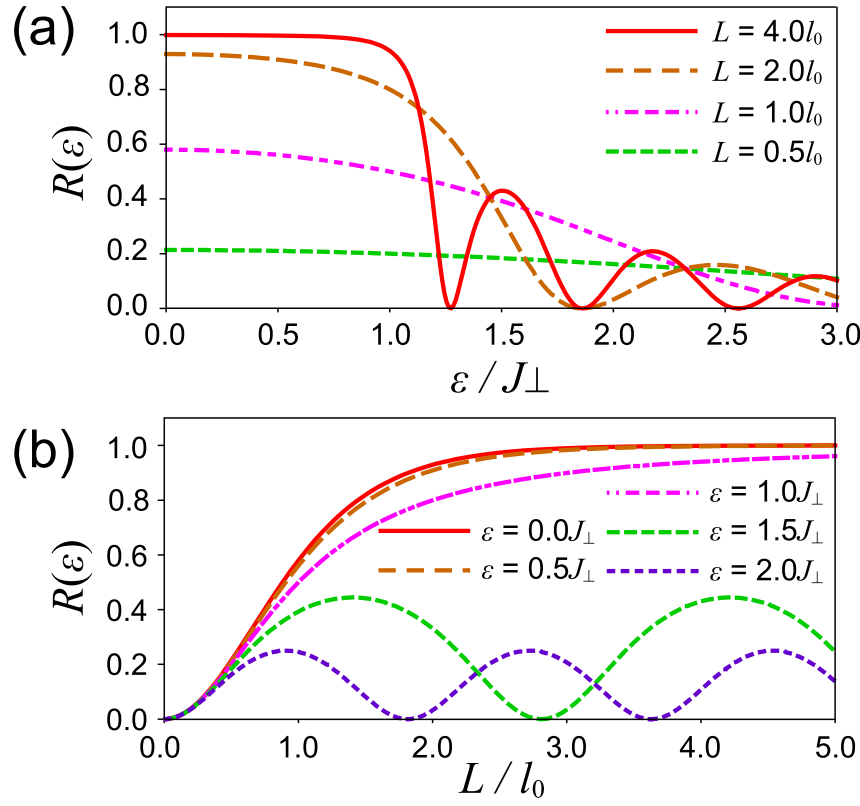

FIG. 4. The reflection rate $R(\epsilon)$ of the magnetic region given by Eq. (21), as a function of (a) the incident energy $\epsilon$ and (b) the length $L$ of the magnetic region. $L$ is rescaled by $l_{0}=v_{\text {edge }} / J_{\perp}$, which is the decay length of the wave function inside the exchange gap of the magnetic region.

assume that the distributions of the incoming channels $f_{\uparrow / \downarrow}^{\text {(in) }}(\epsilon)$ are in equilibrium, with both of them filled up to the Fermi level $\epsilon_{F}$, Eqs. (18) and (19) yield the balance of incoming and outgoing electron numbers,

$$
I_{\uparrow}^{\text {(out) }}-I_{\uparrow}^{\text {(in) }}=I_{\downarrow}^{\text {(in) }}-I_{\downarrow}^{\text {(out) }} \approx \frac{\Omega}{2 \pi} R\left(\epsilon_{F}\right),
$$

up to the first order in $\Omega$ for slow magnetization dynamics. This relation means that the number rate of outgoing electrons with spin $\uparrow$ is raised and that with spin $\downarrow$ is lowered by $R\left(\epsilon_{F}\right) \Omega / 2 \pi$ due to the magnetization dynamics, as schematically shown in Fig. 3 .

In particular, if the Fermi level $\epsilon_{F}$ is inside the exchange gap $\left(\left|\epsilon_{F}\right|<J_{\perp}\right)$, the electrons at the Fermi level are fully reflected, i.e., $R\left(\epsilon_{F}\right) \approx 1$. The right-hand side of Eq. (24) thus reduces to $\Omega / 2 \pi$, which corresponds to one electron per a precession period $T_{p}=2 \pi / \Omega$. As a consequence, the electric current pumped through the magnetic region becomes quantized as

$$
\bar{I}=-e \frac{\Omega}{2 \pi},
$$

which is consistent with the previous literature on the helical edge states of QSHI [53-60]. The spin injection rate (per unit time) from the FMI into the edge electrons is also quantized as

$$
\bar{I}^{s}=\frac{\Omega}{2 \pi},
$$

which satisfies the relation in Eq. (7). The overall contribution from the 2D surface states of TDSM is given by multiplying those quantized values by the factor $v_{z}=2 k_{D} / 2 \pi$. 


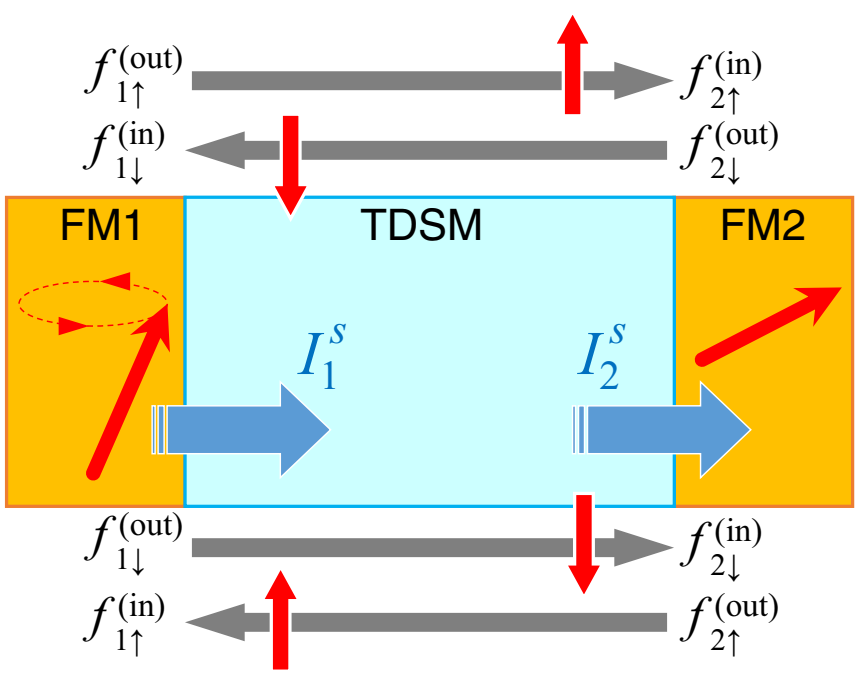

FIG. 5. Schematic picture of the model setup shown in Fig. 1 sliced at a fixed $k_{z}$. On this 2D slice, FM1 and FM2 are connected by four edge channels, two with spin $\uparrow$ and two with spin $\downarrow$. We consider the electron distributions in these channels, to understand the flow of spin $I_{1}^{s}$ and $I_{2}^{s}$.

\section{Spin transfer between two FMIs}

We now consider the model setup constructed in Sec. II. By slicing the system at fixed $k_{z}$ in the band-inverted region $\left(-k_{D}<k_{z}<k_{D}\right)$, FM1 and FM2 are connected by four channels, namely, two pairs of counterpropagating modes with spin $\uparrow$ and $\downarrow$, as shown in Fig. 5. We denote the distribution functions for the incoming or outgoing electrons with spin $s$ $(=\uparrow, \downarrow)$ at $\mathrm{FM} i(i=1,2)$ as $f_{i s}^{\text {(in/out) }}(\epsilon)$. If the electrons on the edges propagate coherently on these channels, $f_{1 \uparrow}^{\text {(out) }}(\epsilon)$ at a certain time is equal to $f_{2 \uparrow}^{(\text {in) }}(\epsilon)$ after a time $T_{x}=L_{x} / v_{\text {edge }}$, and in similar manners for the other channels. Electron propagation on these channels leads to spin transmission between FM1 and FM2. We do not take into account the contribution from the bulk electrons here, which is a valid approximation if the Fermi level is set in the vicinity of the Dirac points so that the density of states in the bulk is small enough.

Now we consider the spin transmission, with the magnetization in FM1 precessing around the $z$ axis by the frequency $\Omega$ and that in FM2 fixed in the $x$ direction. Here, the transmission and reflection coefficients at FM1 are the same as those obtained in Sec. III B (with $L \rightarrow L_{y}$ ), and those at FM2 are given by setting $\Omega=0$. Therefore, in a manner similar to Eqs. (16) and (17), the incoming and outgoing distribution functions are related as follows:

$$
\begin{aligned}
& f_{1 \uparrow}^{\text {(out) }}(\epsilon)=T\left(\epsilon-\frac{\Omega}{2}\right) f_{1 \uparrow}^{\text {(in })}(\epsilon)+R\left(\epsilon-\frac{\Omega}{2}\right) f_{1 \downarrow}^{\text {(in) }}(\epsilon-\Omega), \\
& f_{1 \downarrow}^{\text {(out) }}(\epsilon)=T\left(\epsilon+\frac{\Omega}{2}\right) f_{1 \downarrow}^{\text {(in) }}(\epsilon)+R\left(\epsilon+\frac{\Omega}{2}\right) f_{1 \uparrow}^{\text {(in) }}(\epsilon+\Omega), \\
& f_{2 \uparrow}^{\text {(out) }}(\epsilon)=T(\epsilon) f_{2 \uparrow}^{\text {(in) }}(\epsilon)+R(\epsilon) f_{2 \downarrow}^{\text {(in) }}(\epsilon), \\
& f_{2 \downarrow}^{\text {(out })}(\epsilon)=T(\epsilon) f_{2 \downarrow}^{\text {(in) }}(\epsilon)+R(\epsilon) f_{2 \uparrow}^{\text {(in })}(\epsilon) .
\end{aligned}
$$
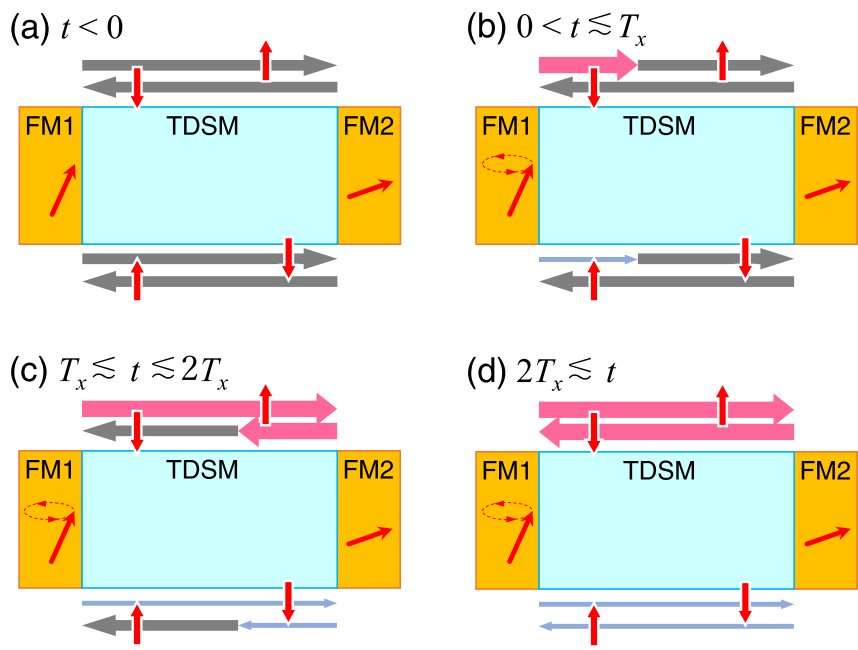

FIG. 6. Schematic pictures of the electron population of edge channels, after switching on the magnetization dynamics in FM1 at time $t=0$. In a manner similar to Fig. 3, gray arrows indicate edge channels in the equilibrium distribution, pink thick arrows indicate channels more populated than the equilibrium distribution, and blue thin arrows indicate less populated channels.

For simplicity of discussion, we assume here that electron transmission and reflection at each magnetic region occur instantaneously, which is satisfied if $L_{y} \ll v_{\text {edge }} T_{p}$.

Based on the above relations, we evaluate the flow of charge and spin between FM1 and FM2, driven by the magnetization dynamics in FM1. As the initial condition, we start with the system in equilibrium, where all the edge channels are in the equilibrium distribution $f_{0}(\epsilon)$ filled up to the Fermi level $\epsilon_{F}$. We then switch on the magnetization dynamics in FM1 at time $t=0$ adiabatically, so that the switch-on process may not cause any significant disturbance in the electron distributions, and estimate the transient behavior of the edge electrons after the switch-on by considering the following steps (a)-(d). (Schematic pictures corresponding to these steps are shown in Fig. 6.)

(a) Before the magnetization dynamics is switched on $(t<0)$, all the edge channels are in the equilibrium distribution $f_{0}(\epsilon)$.

(b) Soon after the switch-on, for time $0 \lesssim t \lesssim T_{x}$, the channels going out from FM1 $\left(f_{1 \uparrow / \downarrow}^{\text {(out) }}\right)$ are modulated by the magnetization dynamics, whereas the incoming channels $\left(f_{1 \uparrow / \downarrow}^{\text {(in) }}\right)$ are still in equilibrium distributions. Therefore $f_{1 \uparrow}^{\text {(out) }}$ and $f_{1 \downarrow}^{\text {(out) }}$ are given as

$$
\begin{aligned}
& f_{1 \uparrow}^{\text {(out) }}(\epsilon)=T\left(\epsilon-\frac{\Omega}{2}\right) f_{0}(\epsilon)+R\left(\epsilon-\frac{\Omega}{2}\right) f_{0}(\epsilon-\Omega), \\
& f_{1 \downarrow}^{\text {(out) }}(\epsilon)=T\left(\epsilon+\frac{\Omega}{2}\right) f_{0}(\epsilon)+R\left(\epsilon+\frac{\Omega}{2}\right) f_{0}(\epsilon+\Omega) .
\end{aligned}
$$

In particular, if the Fermi level $\epsilon_{F}$ is inside the exchange gap and the magnetization dynamics is adiabatic $\left(\Omega \ll J_{\perp}\right)$, we can apply the same discussion as in Eq. (24) in Sec. III B. While the number rates of the incoming electrons per unit time $I_{1 \uparrow}^{\text {(in) }}$ and $I_{1 \downarrow}^{\text {(in) }}$ are equal, the number rate of the outgoing electrons $I_{1 \uparrow}^{\text {(out) }}$ gets raised, and $I_{1 \downarrow}^{\text {(out) }}$ gets lowered by $\Omega / 2 \pi$, due to 
the magnetization dynamics in FM1 [see Fig. 6(b)]. Therefore both the electric current passing through the magnetic region and the spin current pumped from FM1 reach the quantized values

$$
\bar{I}_{1}=-e \frac{\Omega}{2 \pi}, \quad \bar{I}_{1}^{s}=\frac{1}{2} \times \frac{\Omega}{2 \pi}+\frac{-1}{2} \times \frac{-\Omega}{2 \pi}=\frac{\Omega}{2 \pi}
$$

per a single 2D slice at $k_{z}$.

(c) For time $T_{x} \lesssim t \lesssim 2 T_{x}$, the electrons going out from FM1 at step (b) reach FM2 and are reflected or transmitted by FM2. As a consequence, the outgoing distributions for FM1 given by Eqs. (31) and (32) serve as the incoming distributions for FM2, $f_{2 \uparrow}^{\text {(in) }}(\epsilon)=f_{1 \uparrow}^{\text {(out) }}(\epsilon)$ and $f_{2 \downarrow}^{(\text {in })}(\epsilon)=f_{1 \downarrow}^{\text {(in) }}(\epsilon)$. By comparing them with the outgoing distributions $f_{2 \uparrow}^{\text {(out) }}, f_{2 \downarrow}^{\text {(out) }}$ on the basis of the scattering theory in $1 \mathrm{D}$, we see that the spin angular momentum per unit time

$$
I_{2}^{s}=\frac{1 / 2}{2 \pi} \int d \epsilon\left[f_{2 \uparrow}^{(\text {in })}(\epsilon)-f_{2 \downarrow}^{(\text {in })}(\epsilon)-f_{2 \uparrow}^{(\text {out })}(\epsilon)+f_{2 \downarrow}^{\text {(out) }}(\epsilon)\right]
$$

is transferred from the edge electrons to FM2. By substituting Eqs. (29)-(32), this spin current reads

$$
\begin{aligned}
I_{2}^{s}=\frac{1}{2 \pi} \int d \epsilon R(\epsilon)[ & R\left(\epsilon-\frac{\Omega}{2}\right)\left[f_{0}(\epsilon-\Omega)-f_{0}(\epsilon)\right] \\
& -R\left(\epsilon+\frac{\Omega}{2}\right)\left[f_{0}(\epsilon+\Omega)-f_{0}(\epsilon)\right],
\end{aligned}
$$

which is the general form applicable to arbitrary precession frequency $\Omega$ and equilibrium Fermi energy $\epsilon_{F}$.

In particular, if the precession frequency $\Omega$ in FM1 and the Fermi level $\epsilon_{F}$ are inside the exchange gap $J_{\perp}$ (i.e., $\Omega$, $\left.\epsilon_{F} \ll J_{\perp}\right)$, we can again derive the quantized pumping. If the interface region is sufficiently long (i.e., $L_{y} \gg l_{0}$ ), the incoming electrons in the vicinity of $\epsilon_{F}$ are fully reflected at FM2. The incoming electron with spin $\uparrow$, whose number rate per unit time is raised by $\Omega / 2 \pi$, flips its spin on the reflection process at FM2 and injects spin 1 to FM2 for each electron, whereas that with spin $\downarrow$ is lowered by $\Omega / 2 \pi$ and injects spin -1 to FM2 [see Fig. 6(c)]. Therefore the spin current $I_{2}^{s}$ transmitted to FM2 reaches the universal value

$$
\bar{I}_{2}^{s}=1 \times \frac{\Omega}{2 \pi}-1 \times \frac{-\Omega}{2 \pi}=\frac{\Omega}{\pi},
$$

which means that spin 2 is injected into FM2 during a precession period $T_{p}$ per a single 2D slice at $k_{z}$. For the 3D TDSM, the injected spin current (per unit length in the $z$ direction) takes the semiquantized value

$$
\bar{j}_{2}^{s}=v_{z} \bar{I}_{2}^{s}=\frac{k_{D} \Omega}{\pi^{2}} .
$$

This (semi)quantization of spin current is one of the main results in our analysis, which is determined only by the number of helical channels on the surface and is independent of the microscopic structures in the bulk. Note that this relation is satisfied only if the electrons at the Fermi level are fully reflected by FM2. If $\Omega$ or $\epsilon_{F}$ is out of the exchange gap, or once magnetization dynamics is driven in FM2, some elec- trons are transmitted through the magnetic region of FM2, and $I_{2}^{s}$ becomes not exactly twice of $I_{1}^{s}$.

(d) For time $2 T_{x} \lesssim t$, the electrons reflected at FM2 at step (c) reach FM1. At this step, all the edge channels are no longer in equilibrium distribution. Moreover, once the magnetization in FM2 acquires dynamics, the incoming electrons at FM2 are no longer fully reflected. Therefore the spin currents $I_{1}^{S}$ and $I_{2}^{s}$ deviate from the (semi)quantized values $\bar{I}_{1}^{s}$ and $\bar{I}_{2}^{s}$ at this stage. In order to make use of the (semi)quantization of spin current, the magnetization dynamics in FM1 should be in a pulse shorter than the time scale $T_{x}$.

The important feature seen from the analysis above is that the value of the spin current transmitted by the surface states of TDSM [during steps (b) and (c)] is universal and is determined only by the location of the Dirac points $k_{D}$ and the precession frequency $\Omega$, as given by Eq. (37). It only requires the existence of a sizable exchange gap in the helical surface states induced by the FMIs and is insensitive to the microscopic structure and value of the exchange coupling. Moreover, the transmitted spin current is independent of the system size $L_{x, y}$, since only a single pair of helical edge states contribute to the spin transmission for each 2D slice at $k_{z}$. This analytical estimation is valid as long as the surface states are robustly present against disorder, which shall be checked by the numerical simulation in the next section.

\section{NUMERICAL SIMULATION ON A LATTICE}

In this section, we present our numerical simulation of the spin transmission process via a TDSM, which is performed with the 3D lattice model of a TDSM. For the numerical simulation, we use the model constructed in Sec. II, with two FMIs (FM1 and FM2) connected by a TDSM. By following the real-time evolutions of the wave function of all the electrons in the TDSM and of the magnetization in FM2, we evaluate the flow of spin driven by the magnetization dynamics in FM1. As a result, we find that the transmitted spin current reaches the semiquantized value at the early stage after switching on the magnetization dynamics. This semiquantization behavior of the spin current agrees with the surface transport picture employed in the previous section, which implies that the spin transmission in the TDSM is dominated by the surface states. Moreover, we observe that this semiquantized spin transport is robust under disorder even at a long range.

\section{A. Model}

For the numerical simulation, we use a lattice model of a TDSM [36,38]. On a hypothetical cubic lattice with lattice spacing $a$, the tight-binding Hamiltonian

$$
\begin{aligned}
H_{\mathrm{TDSM}}(\boldsymbol{k}) & =u\left[\sin \left(a k_{x}\right) \tau_{x} \sigma_{z}+\sin \left(a k_{y}\right) \tau_{y}\right]-M(\boldsymbol{k}) \tau_{z}, \\
M(\boldsymbol{k}) & =r_{0}-r_{1} \sum_{i=x, y, z}\left[1-\cos \left(a k_{i}\right)\right]
\end{aligned}
$$

reproduces the low-energy effective model in Eq. (1) around $\boldsymbol{k}=0$, with the correspondence of parameters $v=a u, m_{0}=$ $r_{0}$, and $m_{1}=a^{2} r_{1} / 2$. This lattice Hamiltonian gives a pair of Dirac points located at $\boldsymbol{k}_{D}^{ \pm}=\left(0,0, \pm k_{D}\right)$, with $k_{D}=a^{-1}$ $\arccos \left(1-r_{0} / r_{1}\right)$. Throughout our calculation, we fix the parameters $r_{0}=r_{1}=u$, which gives $k_{D}=\pi / 2 a$. 
In real space, the Hamiltonian becomes

$$
\mathcal{H}_{\mathrm{TDSM}}=\sum_{\boldsymbol{r}} \sum_{i=0, x, y, z}\left[c_{\boldsymbol{r}}^{\dagger} D_{i} c_{\boldsymbol{r}+\boldsymbol{a}_{i}}+\text { H.c. }\right]
$$

in the operator formalism, where $c_{\boldsymbol{r}}^{(\dagger)}$ is the four-component annihilation (creation) operator at the lattice site $r$. The vectors $\boldsymbol{a}_{i=0, x, y, z}$ are defined as $\boldsymbol{a}_{0}=0$ and $\boldsymbol{a}_{x, y, z}=a \boldsymbol{e}_{x, y, z}$, with the Cartesian unit vectors $\boldsymbol{e}_{x, y, z}$, and the matrices $D_{i=0, x, y, z}$ are defined as

$$
\begin{aligned}
& D_{0}=-\frac{r_{0}-3 r_{1}}{2} \tau_{z}, \quad D_{z}=-\frac{r_{1}}{2} \tau_{z}, \\
& D_{x}=\frac{i u}{2} \tau_{x} \sigma_{z}-\frac{r_{1}}{2} \tau_{z}, \quad D_{y}=\frac{i u}{2} \tau_{y}-\frac{r_{1}}{2} \tau_{z} .
\end{aligned}
$$

In order to simulate the setup shown in Fig. 1(a), we here take open-boundary conditions in $x$ and $y$ directions, with the size represented by $L_{x}$ and $L_{y}$. For the $z$ direction, we take a periodic boundary condition, with the size $L_{z}$. The number of sites $N_{x, y, z}$ in each direction is related to the system size by $L_{x, y, z}=a N_{x, y, z}$.

The magnetizations in the FMIs are defined as macrospins, with their directions denoted by the unit vectors

$$
\boldsymbol{n}_{i}=\left(\sin \theta_{i} \cos \phi_{i}, \sin \theta_{i} \sin \phi_{i}, \cos \theta_{i}\right) \quad(i=1,2) .
$$

We investigate their dynamics by solving the Landau-LifshitzGilbert (LLG) equation, as we discuss in detail in Sec. IV B, and hence we do not implement their dynamical properties in the Hamiltonian of the TDSM. We require that the magnetizations $\boldsymbol{n}_{1,2}$ are coupled to the electron spins in the TDSM at the boundaries $x=0$ and $x=L_{x}$, respectively. The coupling is described by the Hamiltonian

$$
\mathcal{H}_{\text {exc }}=J_{\text {exc }} \sum_{\boldsymbol{r}}^{x=0} c_{\boldsymbol{r}}^{\dagger}\left(\boldsymbol{n}_{1} \cdot \boldsymbol{\Sigma}\right) c_{\boldsymbol{r}}+J_{\mathrm{exc}} \sum_{\boldsymbol{r}}^{x=L_{x}} c_{\boldsymbol{r}}^{\dagger}\left(\boldsymbol{n}_{2} \cdot \boldsymbol{\Sigma}\right) c_{\boldsymbol{r}},
$$

with the phenomenological coupling constant $J_{\text {exc }}$. The matrix $\Sigma$ characterizes how the exchange coupling depends on the atomic orbital ( $s$ or $p$ ) that each electron in the TDSM belongs to [73]. Here, we define it as $\Sigma=\left(1+\tau_{z}\right) \sigma$, so that the structure of the exchange coupling shall be invariant under a $C_{4}$ rotation around the $z$ axis. By incorporating this coupling term, $\mathcal{H}=\mathcal{H}_{\mathrm{TDSM}}+\mathcal{H}_{\text {exc }}$ is the full Hamiltonian for the electrons in the TDSM.

\section{B. Simulation method}

Based on the lattice model defined above, we perform a numerical simulation of the dynamics of the electrons and the magnetization. The aim of this simulation is to evaluate the influence of the magnetization dynamics in FM1 $\boldsymbol{n}_{1}(t)$ on the magnetization dynamics in FM2 $\boldsymbol{n}_{2}(t)$, which characterizes the spin current transmitted via the TDSM. In order to evaluate them, we simultaneously solve the time-dependent Schrödinger equation

$$
i \partial_{t}|\Psi(t)\rangle=\mathcal{H}(t)|\Psi(t)\rangle
$$

for the many-body wave function $|\Psi(t)\rangle$ for all the electrons in the TDSM [74,75] and the LLG equation

$$
\dot{\boldsymbol{n}}_{2}(t)=-\gamma \boldsymbol{B}_{\text {eff }}(t) \times \boldsymbol{n}_{2}+\alpha \boldsymbol{n}_{2} \times \dot{\boldsymbol{n}}_{2}
$$

for the magnetization in FM2 $\boldsymbol{n}_{2}(t)$, with $\gamma$ being the gyromagnetic ratio and $\alpha$ being the Gilbert damping constant. We introduce the dynamics of $\boldsymbol{n}_{1}(t)$ as the input and do not evaluate the feedback on $\boldsymbol{n}_{1}(t)$ from the electron dynamics. The Hamiltonian $\mathcal{H}(t)$ for the electrons depends on $\boldsymbol{n}_{2}(t)$, and the effective magnetic field $\boldsymbol{B}_{\text {eff }}(t)$ for FM2 depends on $|\Psi(t)\rangle$ via the exchange coupling. In particular, if we define the number and magnitude of spins in FM2 as $N_{s}$ and $S$, the effective magnetic field $\boldsymbol{B}_{\text {eff }}(t)$ for each spin is given by

$$
\begin{aligned}
\gamma \boldsymbol{B}_{\mathrm{eff}}(t) & =-\frac{1}{N_{S} S}\left\langle\frac{\partial \mathcal{H}}{\partial \boldsymbol{n}_{2}}\right\rangle(t) \\
& =-\frac{J_{\mathrm{exc}}}{N_{s} S} \sum_{\boldsymbol{r}}^{x=L_{x}}\left\langle c_{\boldsymbol{r}}^{\dagger} \boldsymbol{\Sigma} c_{\boldsymbol{r}}\right\rangle(t),
\end{aligned}
$$

where $\langle\mathcal{O}\rangle(t)$ denotes the expectation value of the operator $\mathcal{O}$ evaluated with the many-body wave function $|\Psi(t)\rangle$. Equations (43) and (44) are thus correlated, from which we can evaluate the spin-current transmission via the TDSM.

As the initial condition for $t<0$, we set $\boldsymbol{n}_{1}(t<0)=$ $\boldsymbol{n}_{2}(t<0)=\boldsymbol{e}_{x}$ and take $|\Psi(t<0)\rangle$ as the Slater determinant of the occupied states in equilibrium, where all the eigenstates in the TDSM below the Fermi energy $\epsilon_{F}=0$ are occupied. At time $t=0$, we switch on the in-plane magnetization dynamics in FM1

$$
\boldsymbol{n}_{1}(t)=(\cos \Omega t, \sin \Omega t, 0),
$$

with the precession periodicity $T_{p}=2 \pi / \Omega$, and solve the time-dependent equations (43) and (44) simultaneously. In order to evaluate the effect of the transmitted spin current exclusively, we neglect the Gilbert damping $\alpha$ and solve Eq. (44) solely with $\boldsymbol{B}_{\text {eff }}$ from the exchange coupling. We suppose that the spins in FM2 are residing on the lattice sites at the interface $x=L_{x}$, which yields $N_{s}=N_{y} N_{z}$, and fix $S=1$ for the simplicity of calculation. Throughout our simulations, we fix $N_{y}=28$ and $N_{z}=16$.

\section{Spin current versus spin torque}

Before showing our simulation results, we discuss how the spin torque on FM2 calculated from the simulation is related to the spin current flowing into FM2, to compare the simulation results with the analytical estimations given in the previous section. From the torque $\dot{\boldsymbol{n}}_{2}(t)=-\gamma \boldsymbol{B}_{\text {eff }} \times \boldsymbol{n}_{2}$ on FM2, we extract the dampinglike component

$$
\boldsymbol{\tau}_{\mathrm{DL}}(t)=\left(\dot{\boldsymbol{n}}_{2} \cdot \boldsymbol{e}_{2 \theta}\right) \boldsymbol{e}_{2 \theta}(t)=\frac{-\dot{n}_{2 z}(t)}{\sqrt{1-n_{2 z}^{2}}} \boldsymbol{e}_{2 \theta}(t),
$$

where the unit vector $\boldsymbol{e}_{2 \theta}(t)$ is defined by

$$
\begin{aligned}
\boldsymbol{e}_{2 \theta}(t) & =\boldsymbol{n}_{2} \times \frac{\boldsymbol{n}_{2} \times \boldsymbol{e}_{z}}{\left|\boldsymbol{n}_{2} \times \boldsymbol{e}_{z}\right|} \\
& =\left(\cos \theta_{2} \cos \phi_{2}, \cos \theta_{2} \sin \phi_{2},-\sin \theta_{2}\right) .
\end{aligned}
$$

The dampinglike torque tilts the magnetization toward the $z$ axis, which originates from the spin angular momentum injected into the magnet.

We need to check whether the surface-mediated spin current estimated in the previous section is the main contribution to the dampinglike torque $\tau_{\mathrm{DL}}(t)$. From the discussion in 

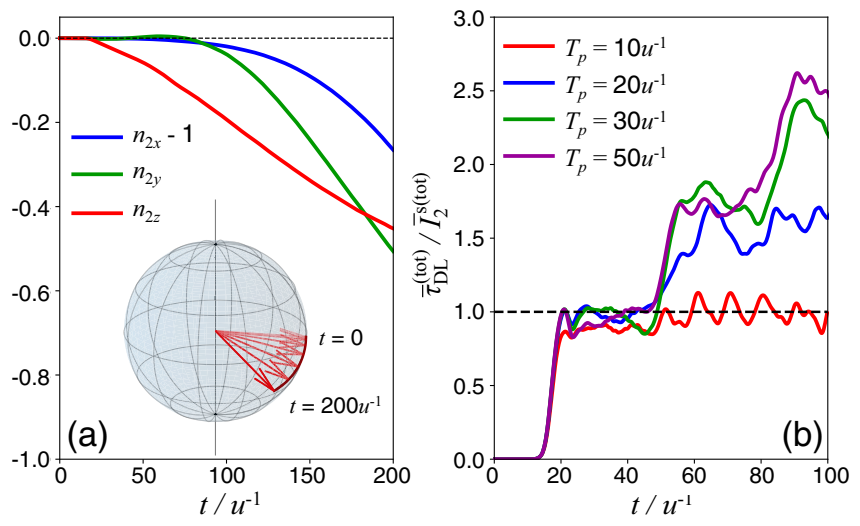

FIG. 7. (a) Time evolution of the components of $\boldsymbol{n}_{2}(t)$ for $J_{\mathrm{exc}}=$ $0.5 u, T_{p}=20 u^{-1}$, and $L_{x}=16 a$. The inset shows the time evolution of $\boldsymbol{n}_{2}(t)$ on the Bloch sphere. (b) Time evolution of the ratio $\rho(t)$ of the dampinglike torque $\tau_{\mathrm{DL}}(t)$ in comparison with $\bar{\tau}_{\mathrm{stt}}(t)$ from the surface transport picture, with several values of $T_{p}$ for $J_{\text {exc }}=0.5 u$ and $L_{x}=16 a$.

the previous section, the net spin current flowing into FM2 reaches the semiquantized value

$$
\bar{I}_{2}^{s(\text { tot })}=L_{z} \bar{j}_{2}^{s}=\frac{L_{z} k_{D} \Omega}{\pi^{2}}
$$

in our lattice system, if the spin transport is dominated by the helical surface states. On the normalized magnetization $\boldsymbol{n}_{2}(t)$ in FM2, this spin current may exert a spin-transfer torque [14]

$$
\begin{aligned}
& \overline{\boldsymbol{\tau}}_{\mathrm{stt}}(t)=\frac{\gamma}{M^{(\mathrm{tot})}} \boldsymbol{n}_{2} \times\left(\boldsymbol{n}_{2} \times \bar{I}_{2}^{s(\text { tot })} \boldsymbol{e}_{z}\right) \\
& =\frac{\sqrt{1-n_{2 z}^{2}}}{N_{s} S} \bar{I}_{2}^{s(\text { tot })} \boldsymbol{e}_{2 \theta}(t) \quad\left(\equiv \bar{\tau}_{\mathrm{stt}} \boldsymbol{e}_{2 \theta}\right),
\end{aligned}
$$

where $M^{(\text {tot })}=\gamma N_{s} S$ denotes the net magnetic moment in FM2.

Therefore, in order to check whether the surface transport picture is valid, we compare the numerically calculated dampinglike torque $\tau_{\mathrm{DL}}(t)$ with this surface-mediated spin-transfer torque $\bar{\tau}_{\text {stt }}(t)$ from estimation, by evaluating their ratio $\rho(t) \equiv$ $\tau_{\mathrm{DL}}(t) / \bar{\tau}_{\mathrm{stt}}(t)$. By using the particular settings $k_{D}=\pi / 2 a$, $N_{s}=N_{y} N_{z}$, and $S=1$ employed in our simulation, this ratio can be derived from the time evolution of $\boldsymbol{n}_{2}(t)$,

$$
\rho(t) \equiv \frac{\tau_{\mathrm{DL}}(t)}{\bar{\tau}_{\mathrm{stt}}(t)}=N_{y} T_{p} \frac{-\dot{n}_{2 z}(t)}{1-n_{2 z}^{2}},
$$

which we shall plot in the following figures. If this ratio $\rho(t)$ reaches unity, we can understand that the spin transmission in the TDSM is dominated by its surface states.

\section{Results and discussion}

We now show our results of the numerical simulations. First, we demonstrate a typical time-evolution behavior of $\boldsymbol{n}_{2}(t)$ in Fig. 7(a). We here take the parameters $J_{\text {exc }}=$ $0.5 u, L_{x}=16 a$, and $T_{p}=20 u^{-1}$. As mentioned above, $\boldsymbol{n}_{2}$ is fixed to the $x$ direction as the initial condition $(t<0)$. After the magnetization dynamics $\boldsymbol{n}_{1}(t)$ in FM1 is switched on at
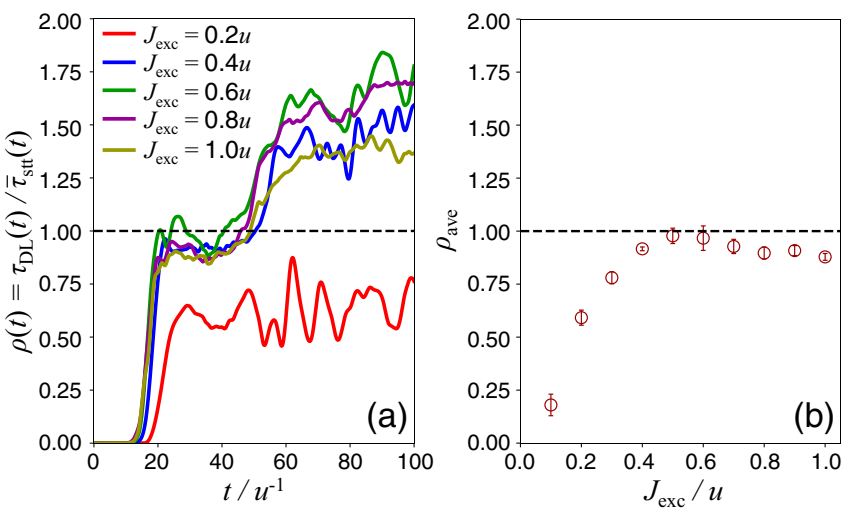

FIG. 8. (a) Time evolution of the ratio $\rho(t)$ of the dampinglike torque $\tau_{\mathrm{DL}}(t)$ in comparison with $\bar{\tau}_{\mathrm{stt}}(t)$ from the surface transport picture, with several values of $J_{\text {exc }}$ for $T_{p}=20 u^{-1}$ and $L_{x}=16 a$. (b) The time-averaged ratio $\rho_{\text {ave }}$ in the window $25 u^{-1}<t<40 u^{-1}$ for several values of $J_{\mathrm{exc}}$, with $T_{p}=20 u^{-1}$ and $L_{x}=16 a$. The error bar on each data point represents the maximum deviation of $\rho(t)$ from the averaged value.

$t=0$, the magnetization $\boldsymbol{n}_{2}(t)$ in FM2 also deviates from its initial direction, which implies that spin angular momentum is transmitted from FM1 to FM2 via the TDSM. We can see that the out-of-plane component $n_{2 z}$ evolves first at the early stage of the magnetization dynamics, which can be considered as the effect of the dampinglike torque from the transmitted spin current.

In order to see the nature of the transmitted spin current in more detail, we plot in Fig. 7(b) the time evolution of $\rho(t)$, namely, the ratio of the dampinglike torque $\tau_{\mathrm{DL}}(t)$ from this simulation in comparison with the spin-transfer torque $\bar{\tau}_{\mathrm{stt}}(t)$ from the surface transport picture, with several values of $T_{p}$. We can see that, for any value of $T_{p}$ in these calculations, $\rho(t)$ reaches unity at the time $t \sim 20 u^{-1}$, which implies that the spin current is dominated by the helical surface states of the TDSM, as predicted in Sec. III C.

The time evolution of the dampinglike torque $\tau_{\mathrm{DL}}^{(\mathrm{tot})}(t)$ can be associated with the transient steps (b)-(d) described in Sec. IIIC (or Fig. 6) as follows. Its zero-value plateau for $t \lesssim u^{-1}$ can be regarded as step (b), with the spin signal from FM1 propagating toward FM2. The semiquantized plateau for $20 u^{-1} \lesssim t \lesssim 40 u^{-1}$ corresponds to step (c), where the signal from FM1 is reflected by FM2 and is injecting spin angular momentum into FM2. For $40 u^{-1} \lesssim t$, the injected spin current deviates from the semiquantized value. This behavior can be associated with step (d), where the signal reflected by FM2 returns to FM1 and gradually enters FM2 again, enhancing the spin injection into FM2.

We next investigate how the transmitted spin current is affected by the exchange coupling parameter $J_{\text {exc }}$ at the interfaces of the TDSM and the FMIs. Figure 8(a) shows the time evolution of the ratio $\rho(t)$ between $\tau_{\mathrm{DL}}(t)$ and $\bar{\tau}_{\mathrm{stt}}(t)$ for several values of $J_{\text {exc }}$, with $T_{p}=20 u^{-1}$. While $\rho(t)$ for $J_{\text {exc }} \gtrsim 0.4 u$ shows a plateau close to unity at the early stage of the magnetization dynamics, the plateau for $J_{\text {exc }}=0.2 u$ is lower than unity. The suppression of the plateau for small $J_{\text {exc }}$ can be clearly seen by plotting the time-averaged 

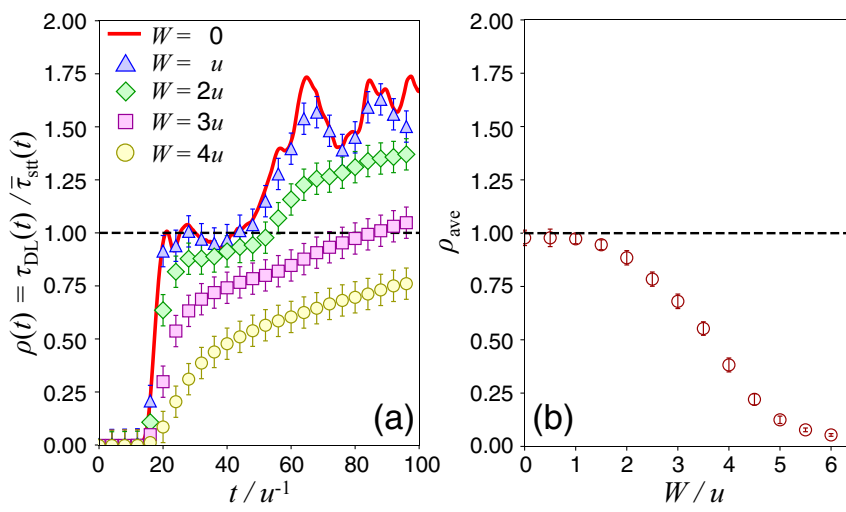

FIG. 9. (a) Time evolution of the ratio $\rho(t)=\tau_{\mathrm{DL}}(t) / \bar{\tau}_{\mathrm{stt}}(t)$ under the random disorder potential for several values of the disorder strength $W$, with $J_{\text {exc }}=0.5 u, T_{p}=20 u^{-1}$, and $L_{x}=16 a$. The error bar on each data point represents the standard error of $\rho(t)$ with respect to the disorder. (b) The time-averaged value $\rho_{\text {ave }}$ of the ratio $\rho(t)$ in the window $25 u^{-1}<t<40 u^{-1}$ for several values of $W$, with $J_{\text {exc }}=0.5 u, T_{p}=20 u^{-1}$, and $L_{x}=16 a$.

value of $\rho(t)$,

$$
\rho_{\mathrm{ave}}=\frac{1}{t_{\mathrm{fin}}-t_{\mathrm{ini}}} \int_{t_{\mathrm{ini}}}^{t_{\mathrm{fin}}} d t \rho(t),
$$

for $t_{\text {ini }}=25 u^{-1}$ and $t_{\text {fin }}=40 u^{-1}$, which is shown in Fig. 8(b). The dependence on $J_{\text {exc }}$ can again be understood from the surface transport picture: The semiquantized spin current is achieved if the magnetization dynamics is adiabatic, which requires the exchange gap $2 J_{\text {exc }}$ on the surface spectrum to be much larger than the precession frequency $\Omega=2 \pi / T_{p} \approx$ $0.3 u^{-1}$ of the magnetization $\boldsymbol{n}_{1}(t)$ (for $T_{p}=20 u^{-1}$ ).

In order to check the robustness of spin transmission against disorder, we introduce the local random potential

$$
\mathcal{H}_{\mathrm{dis}}=\sum_{r} V_{r} c_{r}^{\dagger} c_{r}
$$

where $V_{r}$ takes a random value $V_{r} \in[-W / 2, W / 2]$ for each lattice site $\boldsymbol{r}$, with $W$ characterizing the strength of the disorder. With 20 profiles of the random disorder potential $V_{r}$, we simulate the time evolution of $\boldsymbol{n}_{2}(t)$ and take the average of $\boldsymbol{n}_{2}(t)$ over the 20 profiles to evaluate the disorder-averaged behavior. The time evolution of the ratio $\rho(t)=\tau_{\mathrm{DL}}(t) / \bar{\tau}_{\mathrm{stt}}(t)$ and its time-averaged value $\rho_{\text {ave }}$ in the window $t=25 u^{-1}$ to $40 u^{-1}$ are shown in Figs. 9(a) and 9(b), with $T_{p}=20 u^{-1}$, $J=0.5 u$, and $L_{x}=16 a$. We calculate both the standard errors of the dampinglike torque with respect to the disorder and the disorder-averaged oscillations of the dampinglike torque and plot the larger one as the error bar for each data point. We can see that the plateau $\rho(t) \approx 1$ is achieved for a weak disorder $W \lesssim 2 u$, due to the robustness of the helical surface states under disorder with time-reversal symmetry. The plateau value is gradually suppressed under a strong disorder, once its magnitude exceeds the bandwidth $\sim 2 u$ of the Dirac bands in the bulk.

Finally, in order to check the robustness of the surface spin transport over a long range, we vary the system size $L_{x}$ and observe its effect on the torques. In Fig. 10(a), we show the time evolution of the ratio $\rho(t)=\tau_{\mathrm{DL}}(t) / \bar{\tau}_{\mathrm{stt}}(t)$ for
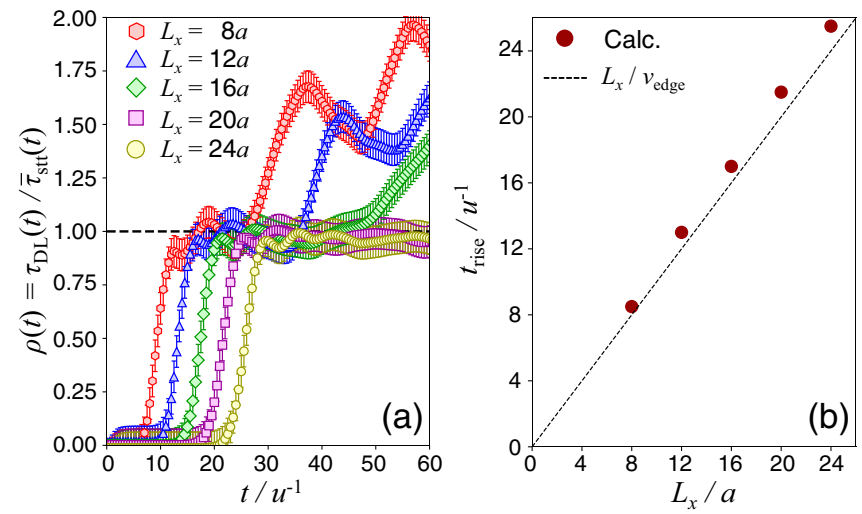

FIG. 10. (a) Time evolution of the ratio $\rho(t)=\tau_{\mathrm{DL}}(t) / \bar{\tau}_{\mathrm{stt}}(t)$ under the random disorder potential of $W=u$ for several values of the system size $L_{x}$, with $J_{\mathrm{exc}}=0.5 u$ and $T_{p}=20 u^{-1}$. The error bar on each data point represents the standard error of $\rho(t)$ with respect to the disorder. (b) The relation between the system size $L_{x}$ and the time $t_{\text {rise }}$ when the calculated ratio $\rho(t)=\tau_{\mathrm{DL}}(t) / \bar{\tau}_{\text {stt }}(t)$ rises to the plateau $\rho(t) \approx 1$. The calculated $t_{\text {rise }}$ agrees well with $t_{\text {rise }}=L_{x} / v_{\text {edge }}$, which comes from the surface transport picture with the velocity $v_{\text {edge }}=u a$.

several values of $L_{x}$, with the disorder strength $W=u$. We can see that $\rho(t)$ rises to the plateau $\approx 1$ at $t_{\text {rise }} \approx L_{x} / v_{\text {edge }}$ (here, $v_{\text {edge }} \approx u a$ ), as shown in Fig. 10(b), namely, the time when an electron propagating from FM1 arrives at FM2. Moreover, the semiquantized plateau is not significantly violated by the disorder, even for a long distance $L_{x}=24 a$. From these results, we can conclude that the helical surface states of the TDSM can realize a long-range spin transport that is robust against a moderate disorder below the bulk bandwidth.

\section{CONCLUSION}

In this paper, we have theoretically demonstrated a longrange spin transport realized by the surface states of a TDSM. TDSMs, such as $\mathrm{Cd}_{3} \mathrm{As}_{2}$ and $\mathrm{Na}_{3} \mathrm{Bi}$, have quasi-1D gapless states on the surface in the form of Fermi arcs, which are spin helical and robust against disorder keeping time-reversal symmetry. By taking a junction of two FMIs and a TDSM as a model setup, as shown in Fig. 1, we have investigated the spin transfer between the two FMIs driven by the magnetization dynamics in one FMI (FM1). We have evaluated the spin transfer both analytically by evaluating the electron numbers in the helical surface channels based on the 1D scattering theory and numerically by simulating the real-time evolution of all the electrons in the TDSM on a lattice model. As a result, we have found that the spin transfer between the two FMIs at charge neutrality is dominated by the helical surface states and that such a surface spin transport is almost insensitive to the disorder keeping time-reversal symmetry.

In particular, at the early stage of the spin transmission after turning on the magnetization dynamics, we have found that the transmitted spin current reaches the semiquantized value $\bar{j}_{2}^{s}$, which is a universal value determined by the precession frequency $\Omega$ of the magnetization dynamics in FM1 and the number of helical channels $v_{z}$ on the surface, corresponding to the distance of the Dirac points $2 k_{D}$ in momentum space. This semiquantized spin current is achieved if the 
magnetization opens a large exchange gap on the helical surface states in comparison with the frequency $\Omega$. This condition is in common with the quantized charge pumping driven by magnetization dynamics on the helical edge states of QSHI [53-61]. Indeed, as discussed in Sec. III, the (semi)quantized charge current and spin current are described in the unified framework based on the edge (surface) transport picture, and they are related independently of the coupling to the FMIs, as in Eq. (6). Since our analysis and simulation show that the transmitted spin current will deviate from the semiquantized value after a long time of magnetization dynamics in FM1, we expect that the semiquantized spin current can be measured if the magnetization dynamics is in a short time, e.g., driven by a microwave pulse.

From our findings in this paper, we expect that the helical surface states of the TDSM are advantageous for long-range spin transport, in comparison with conduction electrons in normal metals or magnons (spin waves) in magnetic insulators. Plus, in comparison with $1 \mathrm{D}$ edge states of 2D topological insulators (QSHIs) and Chern insulators, the helical surface states of 3D TDSMs are advantageous in that they consist of many 1D channels and are capable of transferring a large spin current. The recent transport measurement of a heterostructure of the TDSM $\mathrm{Cd}_{3} \mathrm{As}_{2}$ and a FMI indicates the effect of exchange splitting on the surface states [41], and hence we may expect that the long-range spin transport can be possibly measured with such heterostructures of $\mathrm{Cd}_{3} \mathrm{As}_{2}$.

\section{ACKNOWLEDGMENTS}

This work is supported by JSPS KAKENHI Grant No. 20H01830. Y.A. is supported by the Leading Initiative for Excellent Young Researchers (LEADER). T.M. is supported by JSPS KAKENHI Grants No. JP16H06345 and No. JP19K03739, and by Building of Consortia for the Development of Human Resources in Science and Technology from the MEXT of Japan. K.N. is supported by JST CREST Grant No. JPMJCR18T2.

\section{APPENDIX: $S$ MATRIX}

We here evaluate the scattering rates $R(\epsilon)$ and $T(\epsilon)$ introduced in Sec. III A, which are defined in the rotating frame of spin. Assuming that the FMI is of length $L$ and located at $x_{\|}=0$, the Hamiltonian for the edge electrons in the rest frame is given as

$$
H(t)=v_{\mathrm{edge}} p_{\|} \sigma_{z}+J \boldsymbol{n}(t) \cdot \boldsymbol{\sigma} \pi_{L}\left(x_{\|}\right),
$$

where $\pi_{L}\left(x_{\|}\right)$is the rectangular function taking a value 1 for $-L / 2<x_{\|}<L / 2$ and 0 otherwise, and $p_{\|}=-i \partial / \partial x_{\|}$ is the momentum operator along $x_{\|}$. We take the precession of magnetization around the $z$ axis, where the magnetization direction $\boldsymbol{n}(t)$ is given as

$$
\boldsymbol{n}(t)=(\sin \theta \cos (\Omega t+\phi), \sin \theta \sin (\Omega t+\phi), \cos \theta) .
$$

We introduce $J_{z}=J \cos \theta$ and $J_{\perp}=J \sin \theta$ for later discussions. $J_{\perp}$ gives the exchange gap, if the magnetization is stationary.

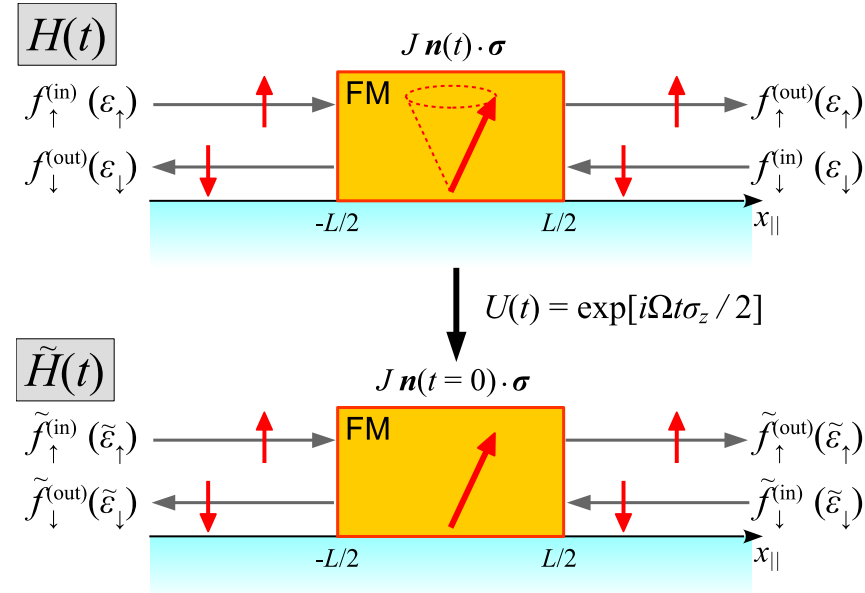

FIG. 11. Schematic pictures for the scattering problem in the rest frame (top) and the rotating frame (bottom). By the unitary transformation $U(t)$ to the rotating frame of spin, the direction of the magnetization is fixed, and the energies of the incoming and outgoing electrons are shifted.

The magnetization $\boldsymbol{n}(t)$ is fixed in the rotating frame of spin. By the time-dependent unitary transformation

$$
U(t)=e^{i \Omega t \sigma_{z} / 2},
$$

which rotates spin by the angular velocity $\Omega$ around the $z$ axis, the Hamiltonian becomes time independent,

$$
\begin{aligned}
\tilde{H} & =U(t)\left[H(t)-i \partial_{t}\right] U^{\dagger}(t) \\
& =\left(v_{\text {edge }} p_{\|}-\frac{\Omega}{2}\right) \sigma_{z}+\operatorname{Jn}(t=0) \cdot \boldsymbol{\sigma} \pi_{L}\left(x_{\|}\right),
\end{aligned}
$$

as schematically shown in Fig. 11. Therefore we can apply the conventional scattering theory with a time-independent scatterer in this rotating frame, to treat the charge and spin pumping by the precessing magnetization. We here fix the energy $\tilde{\epsilon}$ in this frame, evaluate the eigenstate in each region, and connect the obtained eigenstates to derive the scattering solution.

(i) In the nonmagnetic region, the solutions are simply the eigenstates of spin. The plane-wave solutions for rightmoving spin- $\uparrow$ electrons and left-moving spin- $\downarrow$ electrons are given as

$$
\begin{gathered}
e^{i k_{\uparrow} x_{\|}}\left(\begin{array}{l}
1 \\
0
\end{array}\right) \quad\left(v_{\mathrm{F}} k_{\uparrow}=\tilde{\epsilon}+\frac{\Omega}{2}\right), \\
e^{i k_{\downarrow} x_{\|}}\left(\begin{array}{l}
0 \\
1
\end{array}\right) \quad\left(v_{\mathrm{F}} k_{\downarrow}=-\tilde{\epsilon}+\frac{\Omega}{2}\right),
\end{gathered}
$$

respectively.

(ii) The solutions in the magnetic region are given as

$$
e^{i k_{ \pm} x_{\|}} \phi_{\tilde{\epsilon}}^{ \pm}
$$

where the momentum $k_{ \pm}$is defined by

$$
v_{\text {edge }} k_{ \pm}= \pm v_{\text {edge }} K-J_{z}+\frac{\Omega}{2} \quad\left(v_{\text {edge }} K \equiv \sqrt{\tilde{\epsilon}^{2}-J_{\perp}^{2}}\right)
$$


and $\phi_{\tilde{\epsilon}}^{ \pm}$is the eigenvector of the matrix

$$
\begin{aligned}
\left.\tilde{H}\right|_{p_{\|}=k_{ \pm}, \pi_{L}\left(x_{\|}\right)=1} & =\left(\begin{array}{cc}
v_{\text {edge }} k_{ \pm}-\frac{\Omega}{2}+J_{z} & J_{\perp} e^{-i \phi} \\
J_{\perp} e^{i \phi} & -v_{\text {edge }} k_{ \pm}+\frac{\Omega}{2}-J_{z}
\end{array}\right) \\
& =\left(\begin{array}{cc} 
\pm v_{\text {edge }} K & J_{\perp} e^{-i \phi} \\
J_{\perp} e^{i \phi} & \mp v_{\text {edge }} K
\end{array}\right)
\end{aligned}
$$

for the eigenvalue $\tilde{\epsilon}$. In particular, one can write $\phi_{\tilde{\epsilon}}^{ \pm}$as

$$
\phi_{\tilde{\epsilon}}^{+}=\left(\begin{array}{c}
u_{\tilde{\epsilon}} \\
v_{\tilde{\epsilon}} e^{i \phi}
\end{array}\right), \quad \phi_{\tilde{\epsilon}}^{-}=\left(\begin{array}{c}
v_{\tilde{\epsilon}} e^{-i \phi} \\
u_{\tilde{\epsilon}}
\end{array}\right),
$$

with

$$
\begin{gathered}
\left(\begin{array}{c}
u_{\tilde{\epsilon}} \\
v_{\tilde{\epsilon}}
\end{array}\right)=\left(\begin{array}{c}
\tilde{\epsilon}+v_{\text {edge }} K \\
J_{\perp}
\end{array}\right), \quad \text { (A11) The boundary conditions at } x_{\|}= \pm \frac{L}{2} \\
A_{\uparrow} e^{-i k_{\uparrow} L / 2}\left(\begin{array}{l}
1 \\
0
\end{array}\right)+A_{\downarrow} e^{-i k_{\downarrow} L / 2}\left(\begin{array}{c}
0 \\
1
\end{array}\right)=B_{+} e^{-i k_{+} L / 2}\left(\begin{array}{c}
u_{\tilde{\epsilon}} \\
v_{\tilde{\epsilon}} e^{i \phi}
\end{array}\right)+B_{-} e^{-i k_{-} L / 2}\left(\begin{array}{c}
v_{\tilde{\epsilon}} e^{-i \phi} \\
u_{\tilde{\epsilon}}
\end{array}\right), \\
C_{\uparrow} e^{i k_{\uparrow} L / 2}\left(\begin{array}{l}
1 \\
0
\end{array}\right)+C_{\downarrow} e^{i k_{\downarrow} L / 2}\left(\begin{array}{l}
0 \\
1
\end{array}\right)=B_{+} e^{i k_{+} L / 2}\left(\begin{array}{c}
u_{\tilde{\epsilon}} \\
v_{\tilde{\epsilon}} e^{i \phi}
\end{array}\right)+B_{-} e^{i k_{-} L / 2}\left(\begin{array}{c}
v_{\tilde{\epsilon}} e^{-i \phi} \\
u_{\tilde{\epsilon}}
\end{array}\right),
\end{gathered}
$$

which can be assembled into the matrix forms as

$$
\begin{gathered}
\left(\begin{array}{c}
A_{\uparrow} e^{-i k_{\uparrow} L / 2} \\
A_{\downarrow} e^{-i k_{\downarrow} L / 2}
\end{array}\right)=\left(\begin{array}{cc}
u_{\tilde{\epsilon}} e^{-i k_{+} L / 2} & v_{\tilde{\epsilon}} e^{-i \phi-i k_{-} L / 2} \\
v_{\tilde{\epsilon}} e^{i \phi-i k_{+} L / 2} & u_{\tilde{\epsilon}} e^{-i k_{-} L / 2}
\end{array}\right)\left(\begin{array}{l}
B_{+} \\
B_{-}
\end{array}\right), \\
\left(\begin{array}{l}
C_{\uparrow} e^{i k_{\uparrow} L / 2} \\
C_{\downarrow} e^{i k_{\downarrow} L / 2}
\end{array}\right)=\left(\begin{array}{cc}
u_{\tilde{\epsilon}} e^{i k_{+} L / 2} & v_{\tilde{\epsilon}} e^{-i \phi+i k_{-} L / 2} \\
v_{\tilde{\epsilon}} e^{i \phi+i k_{+} L / 2} & u_{\tilde{\epsilon}} e^{i k_{-} L / 2}
\end{array}\right)\left(\begin{array}{l}
B_{+} \\
B_{-}
\end{array}\right) .
\end{gathered}
$$

Therefore the relation between $A_{\uparrow / \downarrow}$ and $C_{\uparrow / \downarrow}$ is given as

$$
\begin{aligned}
\left(\begin{array}{l}
C_{\uparrow} e^{i k_{\uparrow} L / 2} \\
C_{\downarrow} e^{i k_{\downarrow} L / 2}
\end{array}\right) & =\left(\begin{array}{cc}
u_{\tilde{\epsilon}} e^{i k_{+} L / 2} & v_{\tilde{\epsilon}} e^{-i \phi+i k_{-} L / 2} \\
v_{\tilde{\epsilon}} e^{i \phi+i k_{+} L / 2} & u_{\tilde{\epsilon}} e^{i k_{-} L / 2}
\end{array}\right)\left(\begin{array}{cc}
u_{\tilde{\epsilon}} e^{-i k_{+} L / 2} & v_{\tilde{\epsilon}} e^{-i \phi-i k_{-} L / 2} \\
v_{\tilde{\epsilon}} e^{i \phi-i k_{+} L / 2} & u_{\tilde{\epsilon}} e^{-i k_{-} L / 2}
\end{array}\right)^{-1}\left(\begin{array}{c}
A_{\uparrow} e^{-i k_{\uparrow} L / 2} \\
A_{\downarrow} e^{-i k_{\downarrow} L / 2}
\end{array}\right) \\
& =\frac{1}{u_{\tilde{\epsilon}}^{2}-v_{\tilde{\epsilon}}^{2}}\left(\begin{array}{cc}
u_{\tilde{\epsilon}}^{2} e^{i k_{+} L}-v_{\tilde{\epsilon}}^{2} e^{i k_{-} L} & u_{\tilde{\epsilon}} v_{\tilde{\epsilon}} e^{-i \phi}\left(e^{i k_{-} L}-e^{i k_{+} L}\right) \\
u_{\tilde{\epsilon}} v_{\tilde{\epsilon}} e^{i \phi}\left(e^{i k_{+} L}-e^{i k_{-} L}\right) & u_{\tilde{\epsilon}}^{2} e^{i k_{-} L}-v_{\tilde{\epsilon}}^{2} e^{i k_{+} L}
\end{array}\right)\left(\begin{array}{l}
A_{\uparrow} e^{-i k_{\uparrow} L / 2} \\
A_{\downarrow} e^{-i k_{\downarrow} L / 2}
\end{array}\right) .
\end{aligned}
$$

From the definitions $v_{\text {edge }} k_{\uparrow / \downarrow}= \pm \tilde{\epsilon}+\frac{\Omega}{2}$ and $v_{\text {edge }} k_{ \pm}= \pm v_{\text {edge }} K-J_{z}+\frac{\Omega}{2}$, this relation can be further reduced as

$$
\begin{aligned}
\left(\begin{array}{c}
C_{\uparrow} e^{i \tilde{\epsilon} L / 2 v_{\text {edge }}} \\
C_{\downarrow} e^{-i \tilde{\epsilon} L / 2 v_{\text {edge }}}
\end{array}\right) & =\frac{e^{-i J_{z} L / v_{\text {edge }}}}{u_{\tilde{\epsilon}}^{2}-v_{\tilde{\epsilon}}^{2}}\left(\begin{array}{cc}
u_{\tilde{\epsilon}}^{2} e^{i K L}-v_{\tilde{\epsilon}}^{2} e^{-i K L} & u_{\tilde{\epsilon}} v_{\tilde{\epsilon}} e^{-i \phi}\left(e^{-i K L}-e^{i K L}\right) \\
u_{\tilde{\epsilon}} v_{\tilde{\epsilon}} e^{i \phi}\left(e^{i K L}-e^{-i K L}\right) & u_{\tilde{\epsilon}}^{2} e^{-i K L}-v_{\tilde{\epsilon}}^{2} e^{i K L}
\end{array}\right)\left(\begin{array}{c}
A_{\uparrow} e^{-i \tilde{\epsilon} L / 2 v_{\text {edge }}} \\
A_{\downarrow} e^{i \tilde{\epsilon} L / 2 v_{\text {edge }}}
\end{array}\right) \\
& \equiv e^{-i J_{z} L / v_{\text {edge }}}\left(\begin{array}{cc}
\Lambda_{\uparrow \uparrow} & \Lambda_{\uparrow \downarrow} \\
\Lambda_{\downarrow \uparrow} & \Lambda_{\downarrow \downarrow}
\end{array}\right)\left(\begin{array}{c}
A_{\uparrow} e^{-i \tilde{\epsilon} L / 2 v_{\text {edge }}} \\
A_{\downarrow} e^{i \bar{\epsilon} L / 2 v_{\text {edge }}}
\end{array}\right) .
\end{aligned}
$$

Here, we need to recast the above relation into the form of the $S$ matrix,

$$
\left(\begin{array}{c}
C_{\uparrow} \\
A_{\downarrow}
\end{array}\right)=\left(\begin{array}{cc}
\tilde{t}_{\uparrow \uparrow}(\tilde{\epsilon}) & \tilde{r}_{\uparrow \downarrow}(\tilde{\epsilon}) \\
\tilde{r}_{\downarrow \uparrow}(\tilde{\epsilon}) & \tilde{t}_{\downarrow \downarrow}(\tilde{\epsilon})
\end{array}\right)\left(\begin{array}{l}
A_{\uparrow} \\
C_{\downarrow}
\end{array}\right) .
$$

The relation

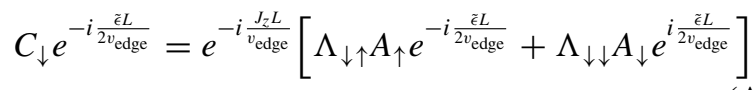

can be rewritten as

$$
A_{\downarrow}=e^{-i \frac{\tilde{\epsilon}}{v_{\text {edge }}} L}\left[-\frac{\Lambda_{\downarrow \uparrow}}{\Lambda_{\downarrow \downarrow}} A_{\uparrow}+\frac{e^{i \frac{J_{z}}{v_{\text {edge }}} L}}{\Lambda_{\downarrow \downarrow}} C_{\downarrow}\right],
$$

which yields

$$
\begin{aligned}
\tilde{r}_{\downarrow \uparrow}(\tilde{\epsilon}) & =-e^{-i \frac{\tilde{\epsilon}}{v_{\text {edge }}} L} \frac{\Lambda_{\downarrow \uparrow}}{\Lambda_{\downarrow \downarrow}} \\
& =-e^{-i\left(\frac{\tilde{\epsilon}}{v_{\text {edge }}} L-\phi\right)} \frac{u_{\tilde{\epsilon}} v_{\tilde{\epsilon}}\left(e^{i K L}-e^{-i K L}\right)}{u_{\tilde{\epsilon}}^{2} e^{-i K L}-v_{\tilde{\epsilon}}^{2} e^{i K L}},
\end{aligned}
$$

$$
\begin{aligned}
\tilde{t}_{\downarrow \downarrow}(\tilde{\epsilon}) & =e^{-i \frac{\tilde{\epsilon}}{v_{\text {edge }}} L} \frac{e^{i \frac{J_{z}}{v_{\text {edge }}} L}}{\Lambda_{\downarrow \downarrow}} \\
& =e^{-i \frac{\tilde{\epsilon}-J_{z}}{v_{\text {edge }}} L} \frac{u_{\tilde{\epsilon}}^{2}-v_{\tilde{\epsilon}}^{2}}{u_{\tilde{\epsilon}}^{2} e^{-i K L}-v_{\tilde{\epsilon}}^{2} e^{i K L}} .
\end{aligned}
$$

The relation

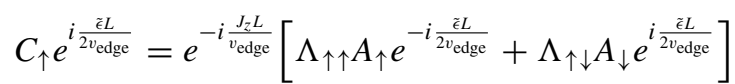


can be rewritten as

$$
\begin{aligned}
C_{\uparrow} & =e^{-i \frac{J_{z}}{v_{\text {edge }}} L}\left[\Lambda_{\uparrow \uparrow} A_{\uparrow} e^{-i \frac{\tilde{\epsilon}}{v_{\text {edge }}} L}+\Lambda_{\uparrow \downarrow} A_{\downarrow}\right] \\
& =e^{-i \frac{J_{z}}{v_{\text {edge }}} L}\left[\Lambda_{\uparrow \uparrow} A_{\uparrow} e^{-i \frac{\tilde{\epsilon}}{v_{\text {edge }}} L}+\Lambda_{\uparrow \downarrow}\left(\tilde{r}_{\downarrow \uparrow} A_{\uparrow}+\tilde{t}_{\downarrow \downarrow} C_{\downarrow}\right)\right],
\end{aligned}
$$

which yields

$$
\begin{aligned}
\tilde{t}_{\uparrow \uparrow}(\tilde{\epsilon}) & =e^{-i \frac{J_{z}}{v_{\text {edge }}} L}\left[\Lambda_{\uparrow \uparrow} e^{-i \frac{\tilde{\epsilon}}{v_{\text {edge }}} L}+\Lambda_{\uparrow \downarrow} \tilde{r}_{\downarrow \uparrow}(\tilde{\epsilon})\right] \\
& =e^{-i \frac{\tilde{\epsilon}+J_{z}}{v_{\text {edge }}} L} \frac{u_{\tilde{\epsilon}}^{2}-v_{\tilde{\epsilon}}^{2}}{u_{\tilde{\epsilon}}^{2} e^{-i K L}-v_{\tilde{\epsilon}}^{2} e^{i K L}}, \\
\tilde{r}_{\uparrow \downarrow}(\tilde{\epsilon}) & =e^{-i \frac{J_{z}}{v_{\text {edge }}} L} \Lambda_{\uparrow \downarrow} \tilde{t}_{\downarrow \downarrow}(\tilde{\epsilon}) \\
& =-e^{-i\left(\frac{\tilde{\epsilon}}{v_{\text {edge }}} L+\phi\right)} \frac{u_{\tilde{\epsilon}} v_{\tilde{\epsilon}}\left(e^{i K L}-e^{-i K L}\right)}{u_{\tilde{\epsilon}}^{2} e^{-i K L}-v_{\tilde{\epsilon}}^{2} e^{i K L}} .
\end{aligned}
$$

The obtained reflection and transmission rates in the rotated frame satisfy the detailed balance relations

$$
\begin{gathered}
\left|\tilde{r}_{\uparrow \downarrow}(\tilde{\epsilon})\right|^{2}=\left|\tilde{r}_{\downarrow \uparrow}(\tilde{\epsilon})\right|^{2}, \\
\left|\tilde{t}_{\uparrow \uparrow}(\tilde{\epsilon})\right|^{2}=\left|\tilde{t}_{\downarrow \downarrow}(\tilde{\epsilon})\right|^{2},
\end{gathered}
$$

which are $R(\tilde{\epsilon})$ and $T(\tilde{\epsilon})$ defined in the main text. From the above calculations, these rates are explicitly obtained as

$$
\begin{aligned}
& R(\tilde{\epsilon})=\frac{1-\cos (2 K L)}{\left(2 \tilde{\epsilon}^{2} / J_{\perp}^{2}\right)-1-\cos (2 K L)}, \\
& T(\tilde{\epsilon})=\frac{\left(2 \tilde{\epsilon}^{2} / J_{\perp}^{2}\right)-2}{\left(2 \tilde{\epsilon}^{2} / J_{\perp}^{2}\right)-1-\cos (2 K L)},
\end{aligned}
$$

where

$$
K=\frac{1}{v_{\text {edge }}} \sqrt{\tilde{\epsilon}^{2}-J_{\perp}^{2}} .
$$

We can check that they satisfy the unitarity condition

$$
R(\tilde{\epsilon})+T(\tilde{\epsilon})=1 .
$$

[1] Spin Physics in Semiconductors, edited by M. I. Dyakonov (Springer, New York, 2008).

[2] S. Takahashi and S. Maekawa, Spin current, spin accumulation and spin Hall effect, Sci. Technol. Adv. Mater. 9, 014105 (2008).

[3] Spin Current, edited by S. Maekawa, S. O. Valenzuela, E. Saitoh, and T. Kimura (Oxford University Press, New York, 2012).

[4] M. Johnson and R. H. Silsbee, Interfacial Charge-Spin Coupling: Injection and Detection of Spin Magnetization in Metals, Phys. Rev. Lett. 55, 1790 (1985).

[5] A. Fert, Two-current conduction in ferromagnetic metals and spin wave-electron collisions, J. Phys. C: Solid State Phys. 2, 1784 (1969).

[6] R. H. Silsbee, A. Janossy, and P. Monod, Coupling between ferromagnetic and conduction-spin-resonance modes at a ferromagnetic-normal-metal interface, Phys. Rev. B 19, 4382 (1979).

[7] Y. Tserkovnyak, A. Brataas, and G. E. W. Bauer, Enhanced Gilbert Damping in Thin Ferromagnetic Films, Phys. Rev. Lett. 88, 117601 (2002).

[8] Y. Tserkovnyak, A. Brataas, G. E. W. Bauer, and B. I. Halperin, Nonlocal magnetization dynamics in ferromagnetic heterostructures, Rev. Mod. Phys. 77, 1375 (2005).

[9] M. I. D'yakonov and V. I. Perel', Possibility of orienting electron spins with current, JETP Lett. 13, 467 (1971).

[10] M. I. Dyakonov and V. I. Perel, Current-induced spin orientation of electrons in semiconductors, Phys. Lett. A 35, 459 (1971).

[11] J. E. Hirsch, Spin Hall Effect, Phys. Rev. Lett. 83, 1834 (1999).

[12] S. Murakami, N. Nagaosa, and S.-C. Zhang, Dissipationless quantum spin current at room temperature, Science 301, 1348 (2003).

[13] J. Sinova, S. O. Valenzuela, J. Wunderlich, C. H. Back, and T. Jungwirth, Spin Hall effects, Rev. Mod. Phys. 87, 1213 (2015).
[14] J. C. Slonczewski, Conductance and exchange coupling of two ferromagnets separated by a tunneling barrier, Phys. Rev. B 39, 6995 (1989).

[15] F. Meier and D. Loss, Magnetization Transport and Quantized Spin Conductance, Phys. Rev. Lett. 90, 167204 (2003).

[16] B. Wang, J. Wang, J. Wang, and D. Y. Xing, Spin current carried by magnons, Phys. Rev. B 69, 174403 (2004).

[17] Y. Kajiwara, K. Harii, S. Takahashi, J. Ohe, K. Uchida, M. Mizuguchi, H. Umezawa, H. Kawai, K. Ando, K. Takanashi, S. Maekawa, and E. Saitoh, Transmission of electrical signals by spin-wave interconversion in a magnetic insulator, Nature (London) 464, 262 (2010).

[18] A. V. Chumak, V. I. Vasyuchka, A. A. Serga, and B. Hillebrands, Magnon spintronics, Nat. Phys. 11, 453 (2015).

[19] C. W. Sandweg, Y. Kajiwara, A. V. Chumak, A. A. Serga, V. I. Vasyuchka, M. B. Jungfleisch, E. Saitoh, and B. Hillebrands, Spin Pumping by Parametrically Excited Exchange Magnons, Phys. Rev. Lett. 106, 216601 (2011).

[20] A. V. Chumak, A. A. Serga, M. B. Jungfleisch, R. Neb, D. A Bozhko, V. S. Tiberkevich, and B. Hillebrands, Direct detection of magnon spin transport by the inverse spin Hall effect, Appl. Phys. Lett. 100, 082405 (2012).

[21] K. Uchida, J. Xiao, H. Adachi, J. Ohe, S. Takahashi, J. Ieda, T. Ota, Y. Kajiwara, H. Umezawa, H. Kawai, G. E. W. Bauer, S. Maekawa, and E. Saitoh, Spin Seebeck insulator, Nat. Mater. 9, 894 (2010).

[22] K. Uchida, H. Adachi, T. Ota, H. Nakayama, S. Maekawa, and E. Saitoh, Observation of longitudinal spin-Seebeck effect in magnetic insulators, Appl. Phys. Lett. 97, 172505 (2010).

[23] L. J. Cornelissen, J. Liu, R. A. Duine, J. B. Youssef, and B. J. van Wees, Long-distance transport of magnon spin information in a magnetic insulator at room temperature, Nat. Phys. 11, 1022 (2015).

[24] L. J. Cornelissen, K. J. H. Peters, G. E. W. Bauer, R. A. Duine, and B. J. van Wees, Magnon spin transport driven 
by the magnon chemical potential in a magnetic insulator, Phys. Rev. B 94, 014412 (2016).

[25] B. L. Giles, Z. Yang, J. S. Jamison, J. M. Gomez-Perez, S. Vélez, L. E. Hueso, F. Casanova, and R. C. Myers, Thermally driven long-range magnon spin currents in yttrium iron garnet due to intrinsic spin Seebeck effect, Phys. Rev. B 96, 180412(R) (2017).

[26] A. Brataas, B. van Wees, O. Klein, G. de Loubens, and M. Viret, Spin insulatronics, Phys. Rep. 885, 1 (2020).

[27] N. Ogawa, W. Koshibae, A. J. Beekman, N. Nagaosa, M. Kubota, M. Kawasaki, and Y. Tokura, Photodrive of magnetic bubbles via magnetoelastic waves, Proc. Natl. Acad. Sci. USA 112, 8977 (2015).

[28] T. Kikkawa, K. Shen, B. Flebus, R. A. Duine, K.-I. Uchida, Z. Qiu, G. E. W. Bauer, and E. Saitoh, Magnon Polarons in the Spin Seebeck Effect, Phys. Rev. Lett. 117, 207203 (2016).

[29] Y. Hashimoto, S. Daimon, R. Iguchi, Y. Oikawa, K. Shen, K. Sato, D. Bossini, Y. Tabuchi, T. Satoh, B. Hillebrands, G. E. W. Bauer, T. H. Johansen, A. Kirilyuk, T. Rasing, and E. Saitoh, All-optical observation and reconstruction of spin wave dispersion, Nat. Commun. 8, 15859 (2017).

[30] S. Streib, H. Keshtgar, and G. E. W. Bauer, Damping of Magnetization Dynamics by Phonon Pumping, Phys. Rev. Lett. 121, 027202 (2018).

[31] A. Rückriegel and R. A. Duine, Long-Range Phonon Spin Transport in Ferromagnet-Nonmagnetic Insulator Heterostructures, Phys. Rev. Lett. 124, 117201 (2020).

[32] K. An, A. N. Litvinenko, R. Kohno, A. A. Fuad, V. V. Naletov, L. Vila, U. Ebels, G. de Loubens, H. Hurdequint, N. Beaulieu, J. Ben Youssef, N. Vukadinovic, G. E. W. Bauer, A. N. Slavin, V. S. Tiberkevich, and O. Klein, Coherent long-range transfer of angular momentum between magnon Kittel modes by phonons, Phys. Rev. B 101, 060407(R) (2020).

[33] B.-J. Yang and N. Nagaosa, Classification of stable threedimensional Dirac semimetals with nontrivial topology, Nat. Commun. 5, 4898 (2014).

[34] A. A. Burkov and Y. B. Kim, $\mathbb{Z}_{2}$ and Chiral Anomalies in Topological Dirac Semimetals, Phys. Rev. Lett. 117, 136602 (2016).

[35] K. Taguchi, D. Oshima, Y. Yamaguchi, T. Hashimoto, Y. Tanaka, and M. Sato, Spin Hall conductivity in topological Dirac semimetals, Phys. Rev. B 101, 235201 (2020).

[36] Z. Wang, Y. Sun, X.-Q. Chen, C. Franchini, G. Xu, H. Weng, $\mathrm{X}$. Dai, and Z. Fang, Dirac semimetal and topological phase transitions in $A_{3} \mathrm{Bi}(A=\mathrm{Na}, \mathrm{K}, \mathrm{Rb})$, Phys. Rev. B 85, 195320 (2012).

[37] Z. K. Liu, B. Zhou, Y. Zhang, Z. J. Wang, H. M. Weng, D. Prabhakaran, S.-K. Mo, Z. X. Shen, Z. Fang, X. Dai, Z. Hussain, and Y. L. Chen, Discovery of a three-dimensional topological Dirac semimetal, $\mathrm{Na}_{3} \mathrm{Bi}$, Science 343, 864 (2014).

[38] Z. Wang, H. Weng, Q. Wu, X. Dai, and Z. Fang, Threedimensional Dirac semimetal and quantum transport in $\mathrm{Cd}_{3} \mathrm{As}_{2}$, Phys. Rev. B 88, 125427 (2013).

[39] M. Neupane, S.-Y. Xu, R. Sankar, N. Alidoust, G. Bian, C. Liu, I. Belopolski, T.-R. Chang, H.-T. Jeng, H. Lin, A. Bansil, F. Chou, and M. Z. Hasan, Observation of a three-dimensional topological Dirac semimetal phase in high-mobility $\mathrm{Cd}_{3} \mathrm{As}_{2}$, Nat. Commun. 5, 3786 (2014).
[40] M. Uchida, Y. Nakazawa, S. Nishihaya, K. Akiba, M. Kriener, Y. Kozuka, A. Miyake, Y. Taguchi, M. Tokunaga, N. Nagaosa, Y. Tokura, and M. Kawasaki, Quantum Hall states observed in thin films of Dirac semimetal $\mathrm{Cd}_{3} \mathrm{As}_{2}$, Nat. Commun. 8, 2274 (2017).

[41] M. Uchida, T. Koretsune, S. Sato, M. Kriener, Y. Nakazawa, S. Nishihaya, Y. Taguchi, R. Arita, and M. Kawasaki, Ferromagnetic state above room temperature in a proximitized topological Dirac semimetal, Phys. Rev. B 100, 245148 (2019).

[42] I. Crassee, R. Sankar, W.-L. Lee, A. Akrap, and M. Orlita, 3D Dirac semimetal $\mathrm{Cd}_{3} \mathrm{As}_{2}$ : A review of material properties, Phys. Rev. Materials 2, 120302 (2018).

[43] T. Liang, Q. Gibson, M. N. Ali, M. Liu, R. J. Cava, and N. P. Ong, Ultrahigh mobility and giant magnetoresistance in the Dirac semimetal $\mathrm{Cd}_{3} \mathrm{As}_{2}$, Nat. Mater. 14, 280 (2015).

[44] E. V. Gorbar, V. A. Miransky, I. A. Shovkovy, and P. O. Sukhachov, Dirac semimetals $A_{3} \mathrm{Bi}(A=\mathrm{Na}, \mathrm{K}, \mathrm{Rb})$ as $\mathbb{Z}_{2}$ Weyl semimetals, Phys. Rev. B 91, 121101(R) (2015).

[45] H. Yi, Z. Wang, C. Chen, Y. Shi, Y. Feng, A. Liang, Z. Xie, S He, J. He, Y. Peng, X. Liu, Y. Liu, L. Zhao, G. Liu, X. Dong, J. Zhang, M. Nakatake, M. Arita, K. Shimada, H. Namatame et al., Evidence of topological surface state in three-dimensional Dirac semimetal $\mathrm{Cd}_{3} \mathrm{As}_{2}$, Sci. Rep. 4, 6106 (2014).

[46] B.-J. Yang, T. Morimoto, and A. Furusaki, Topological charges of three-dimensional Dirac semimetals with rotation symmetry, Phys. Rev. B 92, 165120 (2015).

[47] C. Fang, Y. Chen, H.-Y. Kee, and L. Fu, Topological nodal line semimetals with and without spin-orbital coupling, Phys. Rev. B 92, 081201(R) (2015).

[48] C. L. Kane and E. J. Mele, Quantum Spin Hall Effect in Graphene, Phys. Rev. Lett. 95, 226801 (2005).

[49] B. A. Bernevig and S.-C. Zhang, Quantum Spin Hall Effect, Phys. Rev. Lett. 96, 106802 (2006).

[50] B. A. Bernevig, T. L. Hughes, and S.-C. Zhang, Quantum spin Hall effect and topological phase transition in $\mathrm{HgTe}$ quantum wells, Science 314, 1757 (2006).

[51] S. Nishihaya, M. Uchida, Y. Nakazawa, R. Kurihara, K. Akiba, M. Kriener, A. Miyake, Y. Taguchi, M. Tokunaga, and M. Kawasaki, Quantized surface transport in topological Dirac semimetal films, Nat. Commun. 10, 2564 (2019).

[52] K. Kobayashi and K. Nomura, The ferromagnetic-electrodesinduced Hall effect in topological Dirac semimetals, arXiv:2009.13195.

[53] X.-L. Qi, T. L. Hughes, and S.-C. Zhang, Fractional charge and quantized current in the quantum spin Hall state, Nat. Phys. 4, 273 (2008).

[54] S.-H. Chen, B. K. Nikolić, and C.-R. Chang, Inverse quantum spin Hall effect generated by spin pumping from precessing magnetization into a graphene-based two-dimensional topological insulator, Phys. Rev. B 81, 035428 (2010).

[55] F. Mahfouzi, B. K. Nikolić, S.-H. Chen, and C.-R Chang, Microwave-driven ferromagnet-topological-insulator heterostructures: The prospect for giant spin battery effect and quantized charge pump devices, Phys. Rev. B 82, 195440 (2010).

[56] K. Hattori, Topological pumping of spin-polarized currents through helical edge states due to dynamically generated mass gap, J. Phys. Soc. Jpn. 82, 024708 (2013). 
[57] Q. Meng, S. Vishveshwara, and T. L. Hughes, Spin-transfer torque and electric current in helical edge states in quantum spin Hall devices, Phys. Rev. B 90, 205403 (2014).

[58] W. Y. Deng, W. Luo, H. Geng, M. N. Chen, L. Sheng, and D. Y. Xing, Non-adiabatic topological spin pumping, New J. Phys. 17, 103018 (2015).

[59] M.-J. Wang, J. Wang, and J.-F. Liu, Quantized spin pump on helical edge states of a topological insulator, Sci. Rep. 9, 3378 (2019).

[60] Y. Araki, T. Misawa, and K. Nomura, Dynamical spin-tocharge conversion on the edge of quantum spin Hall insulator, Phys. Rev. Research 2, 023195 (2020).

[61] T. Misawa and K. Nomura, Semi-quantized spin pumping and spin-orbit torques in topological Dirac semimetals, Sci. Rep. 9, 19659 (2019).

[62] A. C. Potter, I. Kimchi, and A. Vishwanath, Quantum oscillations from surface Fermi arcs in Weyl and Dirac semimetals, Nat. Commun. 5, 5161 (2014).

[63] P. J. W. Moll, N. L. Nair, T. Helm, A. C. Potter, I. Kimchi, A. Vishwanath, and J. G. Analytis, Transport evidence for Fermiarc-mediated chirality transfer in the Dirac semimetal $\mathrm{Cd}_{3} \mathrm{As}_{2}$, Nature (London) 535, 266 (2016).

[64] C. Zhang, A. Narayan, S. Lu, J. Zhang, H. Zhang, Z. Ni, X. Yuan, Y. Liu, J.-H. Park, E. Zhang, W. Wang, S. Liu, L. Cheng, L. Pi, Z. Sheng, S. Sanvito, and F. Xiu, Evolution of Weyl orbit and quantum Hall effect in Dirac semimetal $\mathrm{Cd}_{3} \mathrm{As}_{2}$, Nat. Commun. 8, 1272 (2017).

[65] G. Zheng, M. Wu, H. Zhang, W. Chu, W. Gao, J. Lu, Y. Han, J. Yang, H. Du, W. Ning, Y. Zhang, and M. Tian, Recognition of Fermi-arc states through the magnetoresistance quantum oscillations in Dirac semimetal $\mathrm{Cd}_{3} \mathrm{As}_{2}$ nanoplates, Phys. Rev. B 96, 121407(R) (2017).
[66] C. Zhang, Y. Zhang, X. Yuan, S. Lu, J. Zhang, A. Narayan, Y. Liu, H. Zhang, Z. Ni, R. Liu, E. S. Choi, A. Suslov, S. Sanvito, L. Pi, H.-Z. Lu, A. C. Potter, and F. Xiu, Quantum Hall effect based on Weyl orbits in $\mathrm{Cd}_{3} \mathrm{As}_{2}$, Nature (London) 565, 331 (2019).

[67] B.-C. Lin, S. Wang, S. Wiedmann, J.-M. Lu, W.-Z. Zheng, D. Yu, and Z.-M. Liao, Observation of an Odd-Integer Quantum Hall Effect from Topological Surface States in $\mathrm{Cd}_{3} \mathrm{As}_{2}$, Phys. Rev. Lett. 122, 036602 (2019).

[68] R. Landauer, Spatial variation of currents and fields due to localized scatterers in metallic conduction, IBM J. Res. Dev. 1, 223 (1957).

[69] M. Büttiker, Four-Terminal Phase-Coherent Conductance, Phys. Rev. Lett. 57, 1761 (1986).

[70] M. Büttiker, Scattering theory of current and intensity noise correlations in conductors and wave guides, Phys. Rev. B 46, 12485 (1992).

[71] M. Büttiker, H. Thomas, and A. Prêtre, Current partition in multiprobe conductors in the presence of slowly oscillating external potentials, Z. Phys. B: Condens. Matter 94, 133 (1994).

[72] S. Datta, Electronic Transport in Mesoscopic Systems (Cambridge University Press, Cambridge, 1995).

[73] Y. Ominato, S. Tatsumi, and K. Nomura, Spin-orbit crossed susceptibility in topological Dirac semimetals, Phys. Rev. B 99, 085205 (2019).

[74] M. Suzuki, Convergence of general decompositions of exponential operators, Commun. Math. Phys. 163, 491 (1994).

[75] T. Nakanishi, T. Ohtsuki, and T. Kawarabayashi, Dephasing by time-dependent random potentials, J. Phys. Soc. Jpn. 66, 949 (1997). 